\title{
Silicon in the dust formation zone of IRC $+10216^{\star, \star \star}$
}

\author{
L. Decin ${ }^{1,2}$, J. Cernicharo ${ }^{3}$, M. J. Barlow ${ }^{4}$, P. Royer ${ }^{1}$, B. Vandenbussche ${ }^{1}$, R. Wesson ${ }^{4}$, E. T. Polehampton ${ }^{5,6}$, \\ E. De Beck ${ }^{1}$, M. Agúndez ${ }^{3,9}$, J. A. D. L. Blommaert ${ }^{1}$, M. Cohen ${ }^{8}$, F. Daniel ${ }^{3}$, W. De Meester ${ }^{1}$, K. Exter ${ }^{1}$, \\ H. Feuchtgruber ${ }^{10}$, J. P. Fonfría ${ }^{7}$, W. K. Gear ${ }^{11}$, J. R. Goicoechea ${ }^{3}$, H. L. Gomez ${ }^{11}$, M. A. T. Groenewegen ${ }^{12}$, \\ P. C. Hargrave ${ }^{11}$, R. Huygen ${ }^{1}$, P. Imhof ${ }^{13}$, R. J. Ivison ${ }^{14}$, C. Jean ${ }^{1}$, F. Kerschbaum ${ }^{16}$, S. J. Leeks ${ }^{5}$, T. Lim ${ }^{5}$, \\ M. Matsuura ${ }^{4,17}$, G. Olofsson ${ }^{15}$, T. Posch $^{16}$, S. Regibo ${ }^{1}$, G. Savini ${ }^{4}$, B. Sibthorpe ${ }^{14}$, B. M. Swinyard ${ }^{5}$, B. Tercero ${ }^{3}$, \\ C. Waelkens ${ }^{1}$, D. K. Witherick ${ }^{4}$, and J. A. Yates ${ }^{4}$ \\ (Affiliations are available in the online edition)
}

Received 30 March 2010 / Accepted 27 April 2010

\section{ABSTRACT}

\begin{abstract}
The interstellar medium is enriched primarily by matter ejected from evolved low and intermediate mass stars. The outflows from these stars create a circumstellar envelope in which a rich gas-phase and dust-nucleation chemistry takes place. We observed the nearest carbon-rich evolved star, IRC +10216 , using the PACS $(55-210 \mu \mathrm{m})$ and SPIRE $(194-672 \mu \mathrm{m})$ spectrometers on board Herschel. We find several tens of lines from SiS and $\mathrm{SiO}$, including lines from the $v=1$ vibrational level. For SiS these transitions range up to $J=124-123$, corresponding to energies around $6700 \mathrm{~K}$, while the highest detectable transition is $J=90-89$ for $\mathrm{SiO}$, which corresponds to an energy around $8400 \mathrm{~K}$. Both species trace the dust formation zone of IRC +10216 , and the broad energy ranges involved in their detected transitions permit us to derive the physical properties of the gas and the particular zone in which each species has been formed. This allows us to check the accuracy of chemical thermodynamical equilibrium models and the suggested depletion of $\mathrm{SiS}$ and $\mathrm{SiO}$ due to accretion onto dust grains.
\end{abstract}

Key words. techniques: spectroscopic - stars: AGB and post-AGB - stars: carbon - circumstellar matter - stars: mass-loss stars: individual: IRC +10216

\section{Introduction}

IRC +10216 (CW Leo) is the brightest non-Solar System object in the sky at $5 \mu \mathrm{m}$. It is the nearest $(D \sim 150 \mathrm{pc}$, Crosas \& Menten 1997) carbon-rich evolved star, and it serves as an archetype for the study of mass loss on the asymptotic giant branch (AGB). The star is losing mass at a rate of $\sim 1-3 \times 10^{-5} M_{\odot} / \mathrm{yr}$ (Crosas \& Menten 1997; Schöier \& Olofsson 2000), producing a dense, dusty circumstellar envelope (CSE). To date, more than 60 molecules have been detected in the CSE of IRC +10216 (e.g., Cernicharo et al. 2000; He et al. 2008). Thermodynamic equilibrium and non-equilibrium reactions, photochemical reactions, ion-molecule reactions, and the condensation of dust grains establish the abundance stratifications throughout the envelope. It is likely that IRC +10216 is in an advanced evolutionary stage, marking the transition from an AGB to a planetary nebula (Skinner et al. 1998). A detailed study of its infrared emission spectrum can yield unique information on the thermophysical and chemical structure of the outflow and on the history of mass loss during this important evolutionary phase.

\section{Observations and data reduction}

Thanks to its high infrared brightness, IRC +10216 is an ideal target for observation with Herschel (Pilbratt et al. 2010). PACS

\footnotetext{
* Herschel is an ESA space observatory with science instruments provided by European-led Principal Investigator consortia and with important participation from NASA.

$\star \star$ Appendix is only available in electronic form at http: //www . aanda. org
}

and SPIRE spectroscopic observations were obtained in the context of the guaranteed time key programme "Mass-loss of Evolved StarS" (Groenewegen et al., in prep.).

The PACS instrument, its in-orbit performance and calibration, and its scientific capabilities are described in Poglitsch et al. (2010). The PACS spectroscopic observations of IRC +10216 consist of full SED scans between 52 and $210 \mu \mathrm{m}$ obtained in a $3 \times 1$ raster, i.e. a pointing on the central object, and two pointings $30^{\prime \prime}$ either side. The observations were performed on 2009 Nov. 12 (OD 182). The position angle was 110 degrees. The instrument mode was a non-standard version of the chopnod PACS-SED AOT, used with a large chopper throw $\left(6^{\prime}\right)$. The spectral resolving power varies between 1000 and 4500. A description of the observing mode and of the data reduction process can be found in Royer et al. (2010). The only difference with the data reduction of VY CMa as presented in Royer et al. (2010) is that the ground-based calibration was used for IRC +10216 . The estimated calibration uncertainty on the line fluxes is $50 \%$.

The SPIRE FTS measures the Fourier transform of the source spectrum across short (SSW, 194-313 $\mu \mathrm{m}$ ) and long (SLW, 303-671 $\mu \mathrm{m}$ ) wavelength bands simultaneously. The FWHM beamwidths of the SSW and SLW arrays vary between 17-19" and 29-42" respectively. The source spectrum, including the continuum, is restored by taking the inverse transform of the observed interferogram. The absolute flux calibration uncertainty is $15-20 \%$ in the SSW band and $20-30 \%$ in the SLW band above $20 \mathrm{~cm}^{-1}$ (up to $50 \%$ below $20 \mathrm{~cm}^{-1}$ ). For more details on the SPIRE FTS and its calibration see Griffin et al. (2010) and Swinyard et al. (2010). 

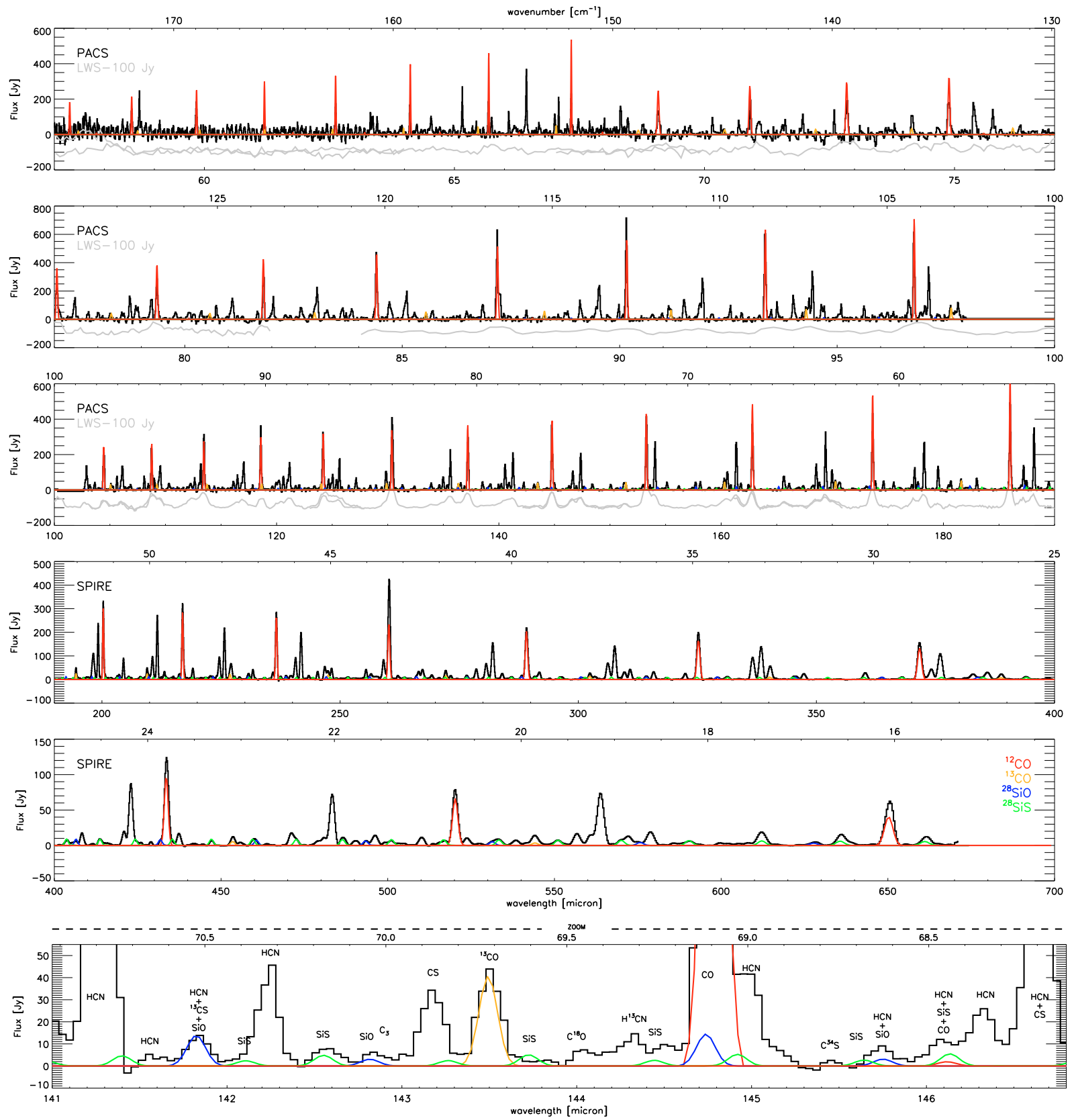

Fig. 1. Continuum-subtracted PACS and SPIRE spectrum of IRC +10216 . In the three upper panels, the PACS spectrum of IRC +10216 (black) is compared to the ISO-LWS spectrum (grey, Cernicharo et al. 1996). The fourth and fifth panels show the SPIRE spectrum of IRC +10216 (black). The bottom panel zooms in on the $141-146.8 \mu \mathrm{m}$ region, where we identified the main molecular features. Theoretical line predictions for ${ }^{12} \mathrm{CO}$ (red), ${ }^{13} \mathrm{CO}$ (orange), ${ }^{28} \mathrm{SiO}$ (blue), and ${ }^{28} \mathrm{SiS}$ (green) using the parameters as given in Table 1 are displayed in all panels.

IRC +10216 was observed with the high-resolution mode of the SPIRE FTS on the 2009 Nov. 19 (OD 189). Twenty repetitions were used, each of which consisted of one forward and one reverse scan of the FTS, with each scan taking $66.6 \mathrm{~s}$. The total on-source integration time was therefore $2664 \mathrm{~s}$. The unapodized spectral resolution is $1.4 \mathrm{GHz}\left(0.048 \mathrm{~cm}^{-1}\right)$, and this is $2.1 \mathrm{GHz}$ $\left(0.07 \mathrm{~cm}^{-1}\right)$ after apodization (using extended Norton-Beer function 1.5; Naylor \& Tahic 2007).

PACS and SPIRE photometry observations of IRC +10216 are presented in Ladjal et al. (2010).

\section{Results}

Currently, more than 500 molecular emission lines have been identified in the PACS and SPIRE spectra of IRC +10216 (see
Fig. 1), belonging to 10 different molecules and their isotopologues $\left({ }^{12} \mathrm{CO},{ }^{13} \mathrm{CO}, \mathrm{C}^{18} \mathrm{O}, \mathrm{H}^{12} \mathrm{CN}, \mathrm{H}^{13} \mathrm{CN}, \mathrm{H}_{2} \mathrm{O}, \mathrm{NH}_{3}, \mathrm{SiS}\right.$, $\mathrm{SiO}, \mathrm{CS}, \mathrm{C}^{34} \mathrm{~S},{ }^{13} \mathrm{CS}, \mathrm{C}_{3}, \mathrm{C}_{2} \mathrm{H}, \mathrm{HCl}$, and $\mathrm{H}^{37} \mathrm{Cl}$ ). The detection of this last molecule is discussed by Cernicharo et al. (2010). In the ISO-LWS spectrum shown in Fig. 1, 57 lines belonging to $\mathrm{CO}$ and HCN were identified by Cernicharo et al. (1996). The number of identified lines increases to 280 in the PACS spectrum thanks to its higher spectral resolution. Most of the lines in the PACS and SPIRE spectrum arise from HCN, with the strongest lines from ${ }^{12} \mathrm{CO}$. $\mathrm{HCN}$ is one of the most abundant molecular species in the CSEs of carbon stars (Willacy \& Cherchneff 1998) and it is known to show maser action in various vibrational states. The strength of the ${ }^{12} \mathrm{CO}$ lines are diagnostics for the thermophysical structure (see Sect. 3.1). In this paper, we 
focus on the silicon-bearing molecules $\mathrm{SiS}$ and $\mathrm{SiO}$, two refractory species that are formed in the inner envelope. As soon as the temperature of the gas falls below a certain critical value, the molecules can start to condense and form dust grains.

High- $J$ rotational lines have been detected from both molecules. For $\mathrm{SiO}, 80$ rotational transitions in the ground-state from $J=11-10$ to $J=90-89\left(E_{\text {up }}=8432 \mathrm{~K}\right)$, and 99 lines from $J=26-25$ to $J=124-123\left(E_{\mathrm{up}}=6678 \mathrm{~K}\right)$ for $\mathrm{SiS}$ are clearly detected. From the detected lines, $\sim 45 \%$ of both species is unblended (see Table A.1 in the online Appendix, which also lists the detected ${ }^{12} \mathrm{CO}$ and ${ }^{13} \mathrm{CO}$ lines). The emission lines of higher- $J$ transitions and rotational transitions in the first vibrational state are very weak, but their line contribution can be deduced from the theoretical modelling (see Sect. 3.2, and Table A.1). The line formation region of the highest- $J$ lines of $\mathrm{SiO}(\mathrm{SiS})$ is within the first $5 R_{\star}\left(10 R_{\star}\right)$, i.e., tracing the recently identified dust formation region (Fonfría et al. 2008).

\subsection{Thermophysical structure of the envelope}

The large number of optically thick ${ }^{12} \mathrm{CO}$ and optically thin ${ }^{13} \mathrm{CO}$ lines enabled us to perform a tomographical study of the CSE. Properties of the circumstellar gas, such as the kinetic temperature, velocity, and density structure, were determined through a non-local thermodynamic equilibrium (non-LTE) radiative transfer modelling of the ${ }^{12} \mathrm{CO}$ lines. The ${ }^{12} \mathrm{CO}$ lines cover energy levels from $J=3$ (at $31 \mathrm{~K}$ ) to $J=47$ (at $5853 \mathrm{~K}$ ) and trace the envelope for radii $R<1 \times 10^{17} \mathrm{~cm}\left(R<2000 R_{\star}\right)$. The GASTRoNOoM code was used to calculate the kinetic temperature and velocity structure in the envelope and to solve the non-LTE radiative transfer equations (Decin et al. 2006, 2010). The rate equations were solved for the ground and first excited vibrational state, with $J_{\max }^{\mathrm{up}}=60$. The $\mathrm{CO}$ line list and collisional rates are discussed in Decin et al. (2010). The terminal velocity was deduced from ground-based observations of low- $J$ ${ }^{12} \mathrm{CO}$ lines (De Beck et al. 2010). The GASTRoNOoM code computes the velocity structure by solving the momentum equation and the temperature structure from the equation expressing the conservation of energy (see Eq. (6) in Decin et al. 2006). However, the resulting temperature was slightly too low beyond $60 R_{\star}$ to correctly predict the lower excitation ${ }^{12} \mathrm{CO}$ lines, which mainly reflects uncertainties in the gas-grain collisional heating. Therefore, we opted to use $T(R) \propto R^{-0.5}$ for $R>60 R_{\star}$.

The best-fit model was determined using the log-likelihood function as described in Decin et al. (2007). The derived (circum)stellar parameters are given in Table 1, the deduced thermodynamical structure is displayed in Fig. 2, and the line predictions are shown in Fig. 1. Specifically, we obtained a mass loss rate of $1 \times 10^{-5} M_{\odot} / y r$ (with an uncertainty of a factor 2 ) and a ${ }^{12} \mathrm{CO} /{ }^{13} \mathrm{CO}$ ratio of $\sim 30 \pm 5$. The latter is on the lower side of the range of ${ }^{12} \mathrm{C} /{ }^{13} \mathrm{C}$ ratios quoted in the literature, going from 20 (Barnes et al. 1977) to 50 (Schöier \& Olofsson 2000). The lowest value is obtained from vibra-rotational transitions in the fundamental band of $\mathrm{CO}$, and higher values are often obtained from low-excitation CO or CS lines. The accuracy of isotopologue ratios obtained from low-excitation rotational transitions is often limited by the uncertain effect of photodissociation by interstellar UV photons and chemical fractionation (e.g., Mamon et al. 1988), effects that are not hampering the high-excitation ${ }^{12} \mathrm{CO}$ and ${ }^{13} \mathrm{CO}$ lines in the PACS and SPIRE spectra.

\subsection{Abundance profiles of $\mathrm{SiO}$ and SiS}

The $\mathrm{SiO}$ and $\mathrm{SiS}$ emission lines are modelled with the thermodynamical structure as deduced in Sect. 3.1. Linelists and
Table 1. Parameters for the best-fit model, where numbers in italics indicate input parameters that have been kept fixed at the given value.

\begin{tabular}{lc|lc}
\hline \hline$T_{\text {eff }}[\mathrm{K}]$ & $2050^{a}$ & $\dot{M}\left[M_{\odot} / \mathrm{yr}\right]$ & $1 \times 10^{-5}$ \\
$R_{\star}\left[10^{13} \mathrm{~cm}\right]$ & 5 & $R_{\text {dust }}\left[R_{\star}\right]$ & $5.6^{d}$ \\
{$\left[\mathrm{CO} / \mathrm{H}_{2}\right]\left[10^{-3}\right]$} & $1^{b}$ & ${ }^{12} \mathrm{CO} /{ }^{13} \mathrm{CO}$ & 30 \\
distance $[\mathrm{pc}]$ & $150^{c}$ & $n(\mathrm{SiO}) / n\left(\mathrm{H}_{2}\right)$ & $1 \times 10^{-7}$ \\
$v_{\infty}\left[\mathrm{km} \mathrm{s}^{-1}\right]$ & 14.5 & $n(\mathrm{SiS}) / n\left(\mathrm{H}_{2}\right)$ & $4 \times 10^{-6}$ \\
\hline
\end{tabular}

References. ${ }^{(a)}$ González-Alfonso et al. (2007), ${ }^{(b)}$ Zuckerman \& Dyck (1986), ${ }^{(c)}$ Crosas \& Menten (1997), ${ }^{(d)}$ Ridgway \& Keady (1988).

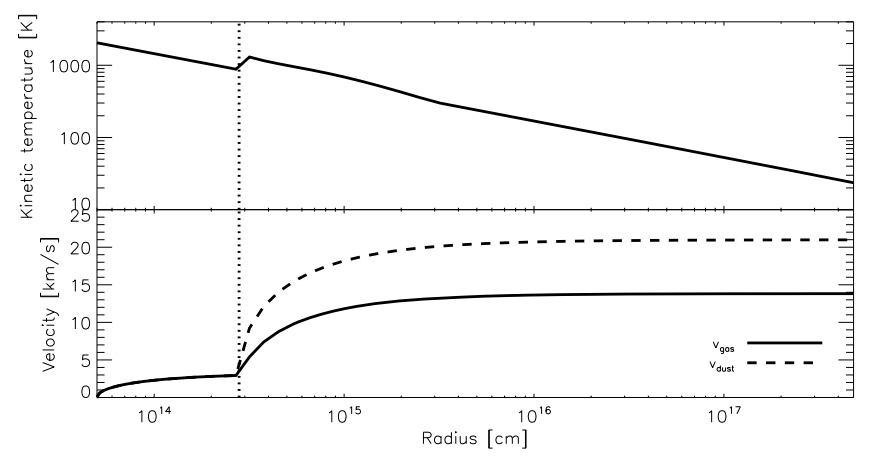

Fig. 2. Thermodynamical structure in the envelope of IRC +10216 as derived from the ${ }^{12} \mathrm{CO}$ rotational line transitions. The vertical dotted line represents the dust condensation radius.

(available) collisional rates are described in Decin et al. (2010). However, the lack of collisional rates for high- $J$ transitions of both molecules with $\mathrm{He}$ or $\mathrm{H}_{2}$ led us calculate the level populations in LTE. This approach is justified since most of the detected high- $J$ lines originate in the stellar photosphere and in the inner wind envelope, where the high gas density and temperature ensure thermal equilibrium for the level populations. Pulsation driven shocks in the inner envelope may alter abundances predicted from equilibrium chemistry. The estimated uncertainty on the derived abundances is a factor of 5, when taking the line flux uncertainty into account.

SiO: Using an outer radius value of $560 R_{\star}$ (Olofsson et al. 1982), the derived fractional abundance is $\left[\mathrm{SiO} / \mathrm{H}_{2}\right]=1 \times 10^{-7}$, when assuming a constant abundance profile. The high- $J \mathrm{SiO}$ lines in the PACS and SPIRE spectrum provide us with a diagnostic tool for deducing possible depletion from the gas from accretion onto dust grains. Unfortunately, the low signal-to-noise ratio of the (weak) high-excitation $\mathrm{SiO}$ lines prohibit us from putting strong constraints on the role of $\mathrm{SiO}$ in the dust formation around IRC +10216 . When allowing for variations in the abundance profile (as described in Decin et al. 2010), we deduce that the $\mathrm{SiO}$ fractional abundance in the inner wind $\left(R \lesssim 8 R_{\star}\right)$ can range between $0.2-3 \times 10^{-7}$, with the fractional abundance being $1 \times 10^{-7}$ beyond $8 R_{\star}$ (see Fig. 3 ). Keady \& Ridgway (1993) derived an inner wind $\mathrm{SiO}$ abundance of $8 \times 10^{-7}$ from infrared ro-vibrational transitions. From low-excitation $\mathrm{SiO}$ lines, Schöier et al. (2006) obtained an $\mathrm{SiO}$ abundance in the region between $\sim 3$ and $8 R_{\star}$, as high as $\sim 1.5 \times 10^{-6}$, superposed on a more spatially extended region of $480 R_{\star}$ with a fractional abundance of $1.7 \times 10^{-7}$. The abundance in this compact inner-wind region is a factor 5 higher than our maximum deduced value of $3 \times 10^{-7}$ in the inner wind. The theoretically calculated photospheric TE value of $\mathrm{SiO}$ in carbon-rich envelopes is $\sim 2.8 \times 10^{-8}$ (Willacy \& Cherchneff 1998). In their study of the effect of 

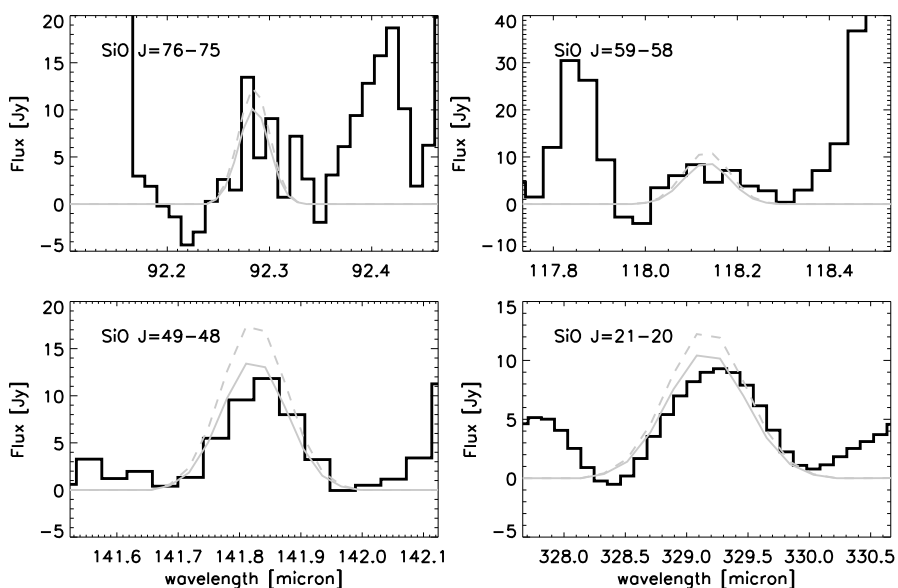

Fig. 3. Comparison between few PACS and SPIRE SiO $v=0$ lines (black) and theoretical line predictions (grey). Full grey lines represent theoretical line profiles using a constant $\mathrm{SiO}$ fractional abundance of $\left[\mathrm{SiO} / \mathrm{H}_{2}\right]=1 \times 10^{-7}$, and dashed grey lines represent model predictions simulating an inner wind abundance of $\left[\mathrm{SiO} / \mathrm{H}_{2}\right]=3 \times 10^{-7}$ for $R<8 R_{\star}$ and $1 \times 10^{-7}$ beyond that radius.

pulsationally induced non-chemical equilibrium in the inner wind of IRC +10216 , Willacy \& Cherchneff (1998) obtained a fractional abundance of $3.8 \times 10^{-7}$. Since TE-value agrees with our minimum deduced value in the inner wind of $2 \times 10^{-8}$, and the non-TE value with the maximum deduced value, the effect of pulsationally induced non-equilibrium chemistry is difficult to estimate.

SiS: The SiS fractional abundances derived from the PACS and SPIRE observations is $\left[\mathrm{SiS} / \mathrm{H}_{2}\right]=4 \times 10^{-6}$. When taking the abundance uncertainty into account, this agrees with the results of Schöier et al. (2007), who find a value of $2 \times 10^{-6}$ from lowexcitation SiS lines. From observations of the $13.5 \mu \mathrm{m}$ fundamental band of SiS, Boyle et al. (1994) obtained a gradient in the abundance of SiS, going from $4.3 \times 10^{-6}$ at a distance of $12 R_{\star}$ and rising to $4.3 \times 10^{-5}$ close to the stellar surface. Bieging $\&$ Nguyen-Quang-Rieu (1989) obtained a much lower value of $\left[\mathrm{SiS} / \mathrm{H}_{2}\right]=7.5 \times 10^{-6}$ for $R<3 \times 10^{15} \mathrm{~cm}$ and $6.5 \times 10^{-7}$ beyond that radius. The PACS and SPIRE observations can allow for a change of a factor of 2 in the first few stellar radii (i.e, minimum of $2 \times 10^{-6}$, maximum of $8 \times 10^{-6}$ for $\left.R \lesssim 12 R_{\star}\right)$. The SiS TE and non-TE inner wind values as computed by Willacy \& Cherchneff $(1998)$ are $\left[\mathrm{SiS} / \mathrm{H}_{2}\right]=1.5 \times 10^{-5}$ and $3.4 \times 10^{-5}$, respectively. That our deduced SiS fractional abundance is clearly lower than the non-TE value of Willacy \& Cherchneff (1998) might indicate toward uncertainties in the estimated rate values in Eqs. (11)-(13) in Willacy \& Cherchneff (1998).

\section{Conclusion}

The PACS and SPIRE spectroscopic observations of IRC +10216 have been shown to be of excellent quality for studying the thermodynamical and chemical structure of the envelope, created by its copious mass loss. The temperature and mass-loss rate of the envelope are derived from the ${ }^{12} \mathrm{CO}$ lines. Both $\mathrm{SiO}$ and $\mathrm{SiS}$ are refractory species, and the PACS and SPIRE data can provide a strong diagnostic tool for determining their role in the dust formation process. Analysing the high- $J$ $\mathrm{SiO}$ and $\mathrm{SiS}$ lines yields a constant fractional abundance of $1 \times 10^{-7}$ and $4 \times 10^{-6}$, respectively. However, we detect only $v=0$ and $v=1$ transitions for both species, mainly because of the densely populated spectrum of IRC +10216 , while it is known from ground-based observations that levels of SiS up to $v=8$ have been detected (Agúndez et al. 2010, in prep.). Moreover, the low- $J$ transitions of $\mathrm{SiO}$ and $\mathrm{SiS}$, which are more sensitive to the external envelope, are not accesible to PACS and SPIRE. Since the high- $J$ lines in the ground-state and the $v=1$ lines of both molecules are very weak, we cannot put strong constraints on the fractional abundance in the inner envelope $\left(R \lesssim 10 R_{\star}\right)$. For $\mathrm{SiO}, 1 / 3$ at most is estimated to take part in dust formation process, while we deduce a fraction of $1 / 2$ for SiS. Only a merged set of millimeter, submillimeter, and far-infrared observations of $\mathrm{SiO}$ and $\mathrm{SiS}$ can provide a detailed analysis of the abundance of these species from the photosphere to the photodissociation zone (Agúndez et al. 2010, in prep.).

Acknowledgements. PACS was developed by a consortium of institutes led by MPE (Germany) and including UVIE (Austria); KUL, CSL, IMEC (Belgium); CEA, OAMP (France); MPIA (Germany); IFSI, OAP/AOT, OAA/CAISMI, LENS, SISSA (Italy); IAC (Spain). This development has been supported by the funding agencies BMVIT (Austria), ESA-PRODEX (Belgium), CEA/CNES (France), DLR (Germany), ASI (Italy), and CICT/MCT (Spain). SPIRE has been developed by a consortium of institutes led by Cardiff Univ. (UK) and including Univ. Lethbridge (Canada); NAOC (China); CEA, LAM (France); IFSI, Univ. Padua (Italy); IAC (Spain); Stockholm Observatory (Sweden); Imperial College London, RAL, UCL-MSSL, UKATC, Univ. Sussex (UK); Caltech, JPL, NHSC, Univ. Colorado (USA). This development has been supported by national funding agencies: CSA (Canada); NAOC (China); CEA, CNES, CNRS (France); ASI (Italy); MCINN (Spain); SNSB (Sweden); STFC (UK); and NASA (USA). L.D. acknowledges financial support from the Fund for Scientific Research Flanders (FWO). M.G., D.L,. J.B., W.D.M., K.E., R.H., C.H., S.R., P.R., and B.V. acknowledge support from the Belgian Federal Science Policy Office via the PRODEX Programme of ESA. F.K. acknowledges funding by the Austrian Science Fund FWF under project number P18939-N16 and I163-N16

\section{References}

Barnes, T. G., Hinkle, K. H., Lambert, D. L., \& Beer, R. 1977, ApJ, 213, 71 Bieging, J. H., \& Nguyen-Quang-Rieu. 1989, ApJ, 343, L25 Boyle, R. J., Keady, J. J., Jennings, D. E., et al. 1994, ApJ, 420, 863 Cernicharo, J., Barlow, M. J., Gonzalez-Alfonso, E., et al. 1996, A\&A, 315, L201

Cernicharo, J., Guélin, M., \& Kahane, C. 2000, A\&AS, 142, 181 Cernicharo, J., Decin, L., Barlow, M., et al. 2010, A\&A, 518, L136 Crosas, M., \& Menten, K. M. 1997, ApJ, 483, 913 De Beck, E., Decin, L., de Koter, A., et al. 2010, A\&A, submitted Decin, L., Hony, S., de Koter, A., et al. 2006, A\&A, 456, 549 Decin, L., Hony, S., de Koter, A., et al. 2007, A\&A, 475, 233 Decin, L., De Beck, E., Brunken, S., et al. 2010, A\&A, 516, A69 Fonfría, J. P., Cernicharo, J., Richter, M. J., \& Lacy, J. H. 2008, ApJ, 673, 445 González-Alfonso, E., Neufeld, D. A., \& Melnick, G. J. 2007, ApJ, 669, 412 Griffin, M. J., Abergel, A., Abreu, A., et al. 2010, A\&A, 518, L3 He, J. H., Dinh-V-Trung, K. S., Müller, H. S. P., et al. 2008, ApJS, 177, 275 Keady, J. J., \& Ridgway, S. T. 1993, ApJ, 406, 199

Ladjal, D., Barlow, M., Groenewegen, M., et al. 2010, A\&A, 518, L141 Mamon, G. A., Glassgold, A. E., \& Huggins, P. J. 1988, ApJ, 328, 797 Naylor, D. A., \& Tahic, M. K. 2007, J. of Optical Soc. of America A, 24, 3644 Olofsson, H., Johansson, L. E. B., Hjalmarson, A., \& Nguyen-Quang-Rieu. 1982, A\&A, 107, 128

Pilbratt, G. L., Riedinger, J. R., Passvogel, T., et al. 2010, A\&A, 518, L1

Poglitsch, A., Waelkens, C., Geis, N., et al. 2010, A\&A, 518, L2 Ridgway, S., \& Keady, J. J. 1988, ApJ, 326, 843

Royer, P., Decin, L., Wesson, R., et al. 2010, A\&A, 518, L145

Schöier, F. L., \& Olofsson, H. 2000, A\&A, 359, 586

Schöier, F. L., Fong, D., Olofsson, H., et al. 2006, ApJ, 649, 965

Schöier, F. L., Bast, J., Olofsson, H., \& Lindqvist, M. 2007, A\&A, 473, 871

Skinner, C. J., Meixner, M., \& Bobrowsky, M. 1998, MNRAS, 300, L29

Swinyard, B. M., Ade, P., Baluteau, J.-P., et al. 2010, A\&A, 518, L4

Willacy, K., \& Cherchneff, I. 1998, A\&A, 330, 676

Zuckerman, B., \& Dyck, H. M. 1986, ApJ, 304, 394

Pages 5 to 11 are available in the electronic edition of the journal at http://www . aanda.org 
1 Instituut voor Sterrenkunde, Katholieke Universiteit Leuven, Celestijnenlaan 200D, 3001 Leuven, Belgium

e-mail: Leen.Decin@ster.kuleuven.be

2 Sterrenkundig Instituut Anton Pannekoek, University of Amsterdam, Science Park 904, 1098 Amsterdam, The Netherlands

${ }^{3}$ Laboratory of Molecular Astrophysics, Department of Astrophysics, CAB, INTA-CSIC, Ctra de Ajalvir, km 4, 28850 Torrejon de Ardoz, Madrid, Spain

4 Dept of Physics \& Astronomy, University College London, Gower St, London WC1E 6BT, UK

5 Space Science and Technology Department, Rutherford Appleton Laboratory, Oxfordshire, OX11 0QX, UK

${ }^{6}$ Department of Physics, University of Lethbridge, Lethbridge, Alberta, T1J 1B1, Canada

7 Departamento de Astrofísica Molecular e Infrarroja, Instituto de Estructura de la Materia, CSIC, Serrano 121, 28006 Madrid, Spain

8 Radio Astronomy Laboratory, University of California at Berkeley, CA 94720, USA
9 LUTH, Observatoire de Paris-Meudon, 5 Place Jules Janssen, 92190 Meudon, France

10 Max-Planck-Institut für extraterrestrische Physik, 85748 Giessenbachstrasse, Germany

11 School of Physics and Astronomy, Cardiff University, Queens Buildings, The Parade, Cardiff, CF24 3AA, UK

12 Royal Observatory of Belgium, Ringlaan 3, 1180 Brussels, Belgium

13 Blue Sky Spectroscopy, 9/740 4 Ave S, Lethbridge, Alberta T1J 0N9, Canada

${ }^{14}$ UK Astronomy Technology Centre, Royal Observatory Edinburgh, Blackford Hill, Edinburgh EH9 3HJ, UK

15 Dept of Astronomy, Stockholm University, AlbaNova University Center, Roslagstullsbacken 21, 10691 Stockholm, Sweden

16 University of Vienna, Department of Astronomy, Türkenschanzstraße 17, 1180 Vienna, Austria

17 Mullard Space Science Laboratory, University College London, Holmbury St. Mary, Dorking, Surrey RH5 6NT, UK 
A\&A 518, L143 (2010)

\section{Appendix A: Identified lines of ${ }^{12} \mathrm{C}^{16} \mathrm{O},{ }^{13} \mathrm{C}^{16} \mathrm{O},{ }^{28} \mathrm{Si}^{16} \mathrm{O}$, and ${ }^{28} \mathrm{Si}^{32} \mathrm{~S}$}

Table A.1. Identified lines of ${ }^{12} \mathrm{C}^{16} \mathrm{O},{ }^{13} \mathrm{C}^{16} \mathrm{O},{ }^{28} \mathrm{Si}^{16} \mathrm{O}$, and ${ }^{28} \mathrm{Si}^{32} \mathrm{~S}$ in the PACS and SPIRE spectrum of IRC 10216.

\begin{tabular}{|c|c|c|c|c|c|}
\hline Molecule & Transition & $\begin{array}{l}\text { Frequency } \\
{[\mathrm{GHz}]}\end{array}$ & $\begin{array}{l}\text { Wavelength } \\
{[\mu \mathrm{m}]}\end{array}$ & $\begin{array}{l}\text { Integrated Flux } \\
{\left[\mathrm{erg} / \mathrm{cm}^{2} / \mathrm{s}\right]}\end{array}$ & Blend \\
\hline${ }^{12} \mathrm{C}^{16} \mathrm{O}$ & $v=0-0, J=4-3$ & 461.042 & 650.250 & $1.35 \mathrm{e}-12$ & no \\
\hline${ }^{12} \mathrm{C}^{16} \mathrm{O}$ & $v=0-0, J=5-4$ & 576.267 & 520.232 & $2.20 \mathrm{e}-12$ & no \\
\hline${ }^{12} \mathrm{C}^{16} \mathrm{O}$ & $v=0-0, J=6-5$ & 691.474 & 433.555 & $3.08 \mathrm{e}-12$ & no \\
\hline${ }^{12} \mathrm{C}^{16} \mathrm{O}$ & $v=0-0, J=7-6$ & 806.652 & 371.650 & $4.17 \mathrm{e}-12$ & no \\
\hline${ }^{12} \mathrm{C}^{16} \mathrm{O}$ & $v=0-0, J=8-7$ & 921.799 & 325.225 & $4.98 \mathrm{e}-12$ & no \\
\hline${ }^{12} \mathrm{C}^{16} \mathrm{O}$ & $v=0-0, J=9-8$ & 1036.913 & 289.120 & $6.01 \mathrm{e}-12$ & no \\
\hline${ }^{12} \mathrm{C}^{16} \mathrm{O}$ & $v=0-0, J=10-9$ & 1151.986 & 260.240 & $6.67 \mathrm{e}-12$ & no \\
\hline${ }^{12} \mathrm{C}^{16} \mathrm{O}$ & $v=0-0, J=11-10$ & 1267.016 & 236.613 & $7.22 \mathrm{e}-12$ & no \\
\hline${ }^{12} \mathrm{C}^{16} \mathrm{O}$ & $v=0-0, J=12-11$ & 1381.995 & 216.927 & $1.98 \mathrm{e}-12$ & no \\
\hline${ }^{12} \mathrm{C}^{16} \mathrm{O}$ & $v=0-0, J=13-12$ & 1496.924 & 200.272 & $2.99 \mathrm{e}-12$ & no \\
\hline${ }^{12} \mathrm{C}^{16} \mathrm{O}$ & $v=0-0, J=14-13$ & 1611.792 & 185.999 & $7.36 \mathrm{e}-12$ & no \\
\hline${ }^{12} \mathrm{C}^{16} \mathrm{O}$ & $v=0-0, J=15-14$ & 1726.604 & 173.631 & $7.31 \mathrm{e}-12$ & no \\
\hline${ }^{12} \mathrm{C}^{16} \mathrm{O}$ & $v=0-0, J=16-15$ & 1841.347 & 162.812 & $7.32 \mathrm{e}-12$ & no \\
\hline${ }^{12} \mathrm{C}^{16} \mathrm{O}$ & $v=0-0, J=17-16$ & 1956.017 & 153.267 & $6.95 \mathrm{e}-12$ & no \\
\hline${ }^{12} \mathrm{C}^{16} \mathrm{O}$ & $v=0-0, J=18-17$ & 2070.616 & 144.784 & $6.68 \mathrm{e}-12$ & no \\
\hline${ }^{12} \mathrm{C}^{16} \mathrm{O}$ & $v=0-0, J=19-18$ & 2185.134 & 137.196 & $6.47 \mathrm{e}-12$ & no \\
\hline${ }^{12} \mathrm{C}^{16} \mathrm{O}$ & $v=0-0, J=20-19$ & 2299.570 & 130.369 & $6.15 \mathrm{e}-12$ & no \\
\hline${ }^{12} \mathrm{C}^{16} \mathrm{O}$ & $v=0-0, J=21-20$ & 2413.917 & 124.193 & $5.88 \mathrm{e}-12$ & no \\
\hline${ }^{12} \mathrm{C}^{16} \mathrm{O}$ & $v=0-0, J=22-21$ & 2528.171 & 118.581 & $5.53 e-12$ & no \\
\hline${ }^{12} \mathrm{C}^{16} \mathrm{O}$ & $v=0-0, J=23-22$ & 2642.332 & 113.458 & $5.08 \mathrm{e}-12$ & no \\
\hline${ }^{12} \mathrm{C}^{16} \mathrm{O}$ & $v=0-0, J=24-23$ & 2756.388 & 108.763 & $4.83 \mathrm{e}-12$ & no \\
\hline${ }^{12} \mathrm{C}^{16} \mathrm{O}$ & $v=0-0, J=25-24$ & 2870.339 & 104.445 & $4.51 \mathrm{e}-12$ & no \\
\hline${ }^{12} \mathrm{C}^{16} \mathrm{O}$ & $v=0-0, J=27-26$ & 3097.909 & 96.773 & $4.06 \mathrm{e}-12$ & no \\
\hline${ }^{12} \mathrm{C}^{16} \mathrm{O}$ & $v=0-0, J=28-27$ & 3211.518 & 93.349 & $3.85 \mathrm{e}-12$ & no \\
\hline${ }^{12} \mathrm{C}^{16} \mathrm{O}$ & $v=0-0, J=29-28$ & 3325.005 & 90.163 & $3.55 \mathrm{e}-12$ & yes \\
\hline${ }^{12} \mathrm{C}^{16} \mathrm{O}$ & $v=0-0, J=30-29$ & 3438.365 & 87.190 & $3.38 \mathrm{e}-12$ & yes \\
\hline${ }^{12} \mathrm{C}^{16} \mathrm{O}$ & $v=0-0, J=31-30$ & 3551.594 & 84.411 & $3.09 \mathrm{e}-12$ & no \\
\hline${ }^{12} \mathrm{C}^{16} \mathrm{O}$ & $v=0-0, J=32-31$ & 3664.685 & 81.806 & $2.95 \mathrm{e}-12$ & no \\
\hline${ }^{12} \mathrm{C}^{16} \mathrm{O}$ & $v=0-0, J=33-32$ & 3777.637 & 79.360 & $2.68 \mathrm{e}-12$ & no \\
\hline${ }^{12} \mathrm{C}^{16} \mathrm{O}$ & $v=0-0, J=34-33$ & 3890.443 & 77.059 & $2.59 \mathrm{e}-12$ & no \\
\hline${ }^{12} \mathrm{C}^{16} \mathrm{O}$ & $v=0-0, J=35-34$ & 4003.102 & 74.890 & $2.31 \mathrm{e}-12$ & no \\
\hline${ }^{12} \mathrm{C}^{16} \mathrm{O}$ & $v=0-0, J=36-35$ & 4115.605 & 72.843 & $2.18 \mathrm{e}-12$ & no \\
\hline${ }^{12} \mathrm{C}^{16} \mathrm{O}$ & $v=0-0, J=37-36$ & 4227.953 & 70.907 & $2.05 \mathrm{e}-12$ & yes \\
\hline${ }^{12} \mathrm{C}^{16} \mathrm{O}$ & $v=0-0, J=38-37$ & 4340.138 & 69.074 & $1.89 \mathrm{e}-12$ & no \\
\hline${ }^{12} \mathrm{C}^{16} \mathrm{O}$ & $v=0-0, J=39-38$ & 4452.159 & 67.336 & $1.74 \mathrm{e}-12$ & no \\
\hline${ }^{12} \mathrm{C}^{16} \mathrm{O}$ & $v=0-0, J=40-39$ & 4564.005 & 65.686 & $1.60 \mathrm{e}-12$ & no \\
\hline${ }^{12} \mathrm{C}^{16} \mathrm{O}$ & $v=0-0, J=41-40$ & 4675.681 & 64.117 & $1.50 \mathrm{e}-12$ & no \\
\hline${ }^{12} \mathrm{C}^{16} \mathrm{O}$ & $v=0-0, J=42-41$ & 4787.174 & 62.624 & $1.34 \mathrm{e}-12$ & no \\
\hline${ }^{12} \mathrm{C}^{16} \mathrm{O}$ & $v=0-0, J=43-42$ & 4898.484 & 61.201 & $1.31 \mathrm{e}-12$ & no \\
\hline${ }^{12} \mathrm{C}^{16} \mathrm{O}$ & $v=0-0, J=44-43$ & 5009.608 & 59.843 & $1.18 \mathrm{e}-12$ & yes \\
\hline${ }^{12} \mathrm{C}^{16} \mathrm{O}$ & $v=0-0, J=45-44$ & 5120.540 & 58.547 & $1.12 \mathrm{e}-12$ & no \\
\hline${ }^{12} \mathrm{C}^{16} \mathrm{O}$ & $v=1-1, J=28-27$ & 3182.136 & 94.211 & $1.42 \mathrm{e}-13$ & yes \\
\hline${ }^{12} \mathrm{C}^{16} \mathrm{O}$ & $v=1-1, J=29-28$ & 3294.573 & 90.996 & $1.41 \mathrm{e}-13$ & yes \\
\hline${ }^{12} \mathrm{C}^{16} \mathrm{O}$ & $v=1-1, J=30-29$ & 3406.884 & 87.996 & $1.51 \mathrm{e}-13$ & no \\
\hline${ }^{12} \mathrm{C}^{16} \mathrm{O}$ & $v=1-1, J=31-30$ & 3519.060 & 85.191 & $1.48 \mathrm{e}-13$ & no \\
\hline${ }^{12} \mathrm{C}^{16} \mathrm{O}$ & $v=1-1, J=32-31$ & 3631.105 & 82.562 & $1.51 \mathrm{e}-13$ & no \\
\hline${ }^{13} \mathrm{C}^{16} \mathrm{O}$ & $v=0-0, J=5-4$ & 550.926 & 544.161 & $1.17 \mathrm{e}-13$ & yes \\
\hline${ }^{13} \mathrm{C}^{16} \mathrm{O}$ & $v=0-0, J=6-5$ & 661.066 & 453.498 & $1.75 \mathrm{e}-13$ & no \\
\hline${ }^{13} \mathrm{C}^{16} \mathrm{O}$ & $v=0-0, J=7-6$ & 771.183 & 388.743 & $2.39 \mathrm{e}-13$ & yes \\
\hline${ }^{13} \mathrm{C}^{16} \mathrm{O}$ & $v=0-0, J=8-7$ & 881.273 & 340.181 & $3.19 \mathrm{e}-13$ & yes \\
\hline${ }^{13} \mathrm{C}^{16} \mathrm{O}$ & $v=0-0, J=9-8$ & 991.330 & 302.414 & $4.05 \mathrm{e}-13$ & no \\
\hline${ }^{13} \mathrm{C}^{16} \mathrm{O}$ & $v=0-0, J=10-9$ & 1101.351 & 272.204 & $4.85 e-13$ & no \\
\hline${ }^{13} \mathrm{C}^{16} \mathrm{O}$ & $v=0-0, J=11-10$ & 1211.330 & 247.490 & $5.39 \mathrm{e}-13$ & no \\
\hline
\end{tabular}


Table A.1. continued.

\begin{tabular}{|c|c|c|c|c|c|}
\hline Molecule & Transition & $\begin{array}{l}\text { Frequency } \\
{[\mathrm{GHz}]}\end{array}$ & $\begin{array}{l}\text { Wavelength } \\
{[\mu \mathrm{m}]}\end{array}$ & $\begin{array}{l}\text { Integrated Flux } \\
{\left[\mathrm{erg} / \mathrm{cm}^{2} / \mathrm{s}\right]}\end{array}$ & Blend \\
\hline${ }^{13} \mathrm{C}^{16} \mathrm{O}$ & $v=0-0, J=12-11$ & 1321.267 & 226.898 & $6.11 \mathrm{e}-13$ & yes \\
\hline${ }^{13} \mathrm{C}^{16} \mathrm{O}$ & $v=0-0, J=13-12$ & 1431.152 & 209.476 & $6.27 \mathrm{e}-13$ & no \\
\hline${ }^{13} \mathrm{C}^{16} \mathrm{O}$ & $v=0-0, J=14-13$ & 1540.987 & 194.546 & $2.91 \mathrm{e}-13$ & no \\
\hline${ }^{13} \mathrm{C}^{16} \mathrm{O}$ & $v=0-0, J=15-14$ & 1650.768 & 181.608 & $6.94 \mathrm{e}-13$ & no \\
\hline${ }^{13} \mathrm{C}^{16} \mathrm{O}$ & $v=0-0, J=16-15$ & 1760.487 & 170.290 & $6.99 \mathrm{e}-13$ & no \\
\hline${ }^{13} \mathrm{C}^{16} \mathrm{O}$ & $v=0-0, J=17-16$ & 1870.142 & 160.305 & $7.05 \mathrm{e}-13$ & no \\
\hline${ }^{13} \mathrm{C}^{16} \mathrm{O}$ & $v=0-0, J=18-17$ & 1979.728 & 151.431 & $7.03 \mathrm{e}-13$ & no \\
\hline${ }^{13} \mathrm{C}^{16} \mathrm{O}$ & $v=0-0, J=19-18$ & 2089.239 & 143.494 & $6.87 e-13$ & no \\
\hline${ }^{13} \mathrm{C}^{16} \mathrm{O}$ & $v=0-0, J=20-19$ & 2198.678 & 136.351 & $6.68 \mathrm{e}-13$ & no \\
\hline${ }^{13} \mathrm{C}^{16} \mathrm{O}$ & $v=0-0, J=21-20$ & 2308.034 & 129.891 & $6.32 \mathrm{e}-13$ & yes \\
\hline${ }^{13} \mathrm{C}^{16} \mathrm{O}$ & $v=0-0, J=22-21$ & 2417.308 & 124.019 & $6.36 \mathrm{e}-13$ & yes \\
\hline${ }^{13} \mathrm{C}^{16} \mathrm{O}$ & $v=0-0, J=23-22$ & 2526.492 & 118.660 & $5.93 e-13$ & yes \\
\hline${ }^{13} \mathrm{C}^{16} \mathrm{O}$ & $v=0-0, J=24-23$ & 2635.584 & 113.748 & $5.73 e-13$ & no \\
\hline${ }^{13} \mathrm{C}^{16} \mathrm{O}$ & $v=0-0, J=25-24$ & 2744.579 & 109.231 & $5.63 e-13$ & no \\
\hline${ }^{13} \mathrm{C}^{16} \mathrm{O}$ & $v=0-0, J=26-25$ & 2853.476 & 105.062 & $5.39 \mathrm{e}-13$ & no \\
\hline${ }^{13} \mathrm{C}^{16} \mathrm{O}$ & $v=0-0, J=28-27$ & 3070.949 & 97.622 & $4.90 \mathrm{e}-13$ & no \\
\hline${ }^{13} \mathrm{C}^{16} \mathrm{O}$ & $v=0-0, J=29-28$ & 3179.518 & 94.289 & $4.75 e-13$ & no \\
\hline${ }^{13} \mathrm{C}^{16} \mathrm{O}$ & $v=0-0, J=30-29$ & 3287.974 & 91.178 & $4.58 \mathrm{e}-13$ & no \\
\hline${ }^{13} \mathrm{C}^{16} \mathrm{O}$ & $v=0-0, J=31-30$ & 3396.307 & 88.270 & $4.39 \mathrm{e}-13$ & no \\
\hline${ }^{13} \mathrm{C}^{16} \mathrm{O}$ & $v=0-0, J=32-31$ & 3504.517 & 85.545 & $4.22 \mathrm{e}-13$ & no \\
\hline${ }^{13} \mathrm{C}^{16} \mathrm{O}$ & $v=0-0, J=33-32$ & 3612.599 & 82.985 & $4.11 \mathrm{e}-13$ & yes \\
\hline${ }^{13} \mathrm{C}^{16} \mathrm{O}$ & $v=0-0, J=34-33$ & 3720.548 & 80.578 & $3.97 e-13$ & no \\
\hline${ }^{13} \mathrm{C}^{16} \mathrm{O}$ & $v=0-0, J=35-34$ & 3828.359 & 78.308 & $3.83 e-13$ & no \\
\hline${ }^{13} \mathrm{C}^{16} \mathrm{O}$ & $v=0-0, J=36-35$ & 3936.033 & 76.166 & $3.72 \mathrm{e}-13$ & no \\
\hline${ }^{13} \mathrm{C}^{16} \mathrm{O}$ & $v=0-0, J=37-36$ & 4043.562 & 74.141 & $3.63 e-13$ & yes \\
\hline${ }^{13} \mathrm{C}^{16} \mathrm{O}$ & $v=0-0, J=38-37$ & 4150.945 & 72.223 & $3.54 \mathrm{e}-13$ & yes \\
\hline${ }^{13} \mathrm{C}^{16} \mathrm{O}$ & $v=0-0, J=39-38$ & 4258.172 & 70.404 & $3.52 \mathrm{e}-13$ & no \\
\hline${ }^{13} \mathrm{C}^{16} \mathrm{O}$ & $v=0-0, J=40-39$ & 4365.246 & 68.677 & $3.35 \mathrm{e}-13$ & no \\
\hline${ }^{13} \mathrm{C}^{16} \mathrm{O}$ & $v=0-0, J=41-40$ & 4472.161 & 67.035 & $3.33 \mathrm{e}-13$ & no \\
\hline${ }^{13} \mathrm{C}^{16} \mathrm{O}$ & $v=0-0, J=42-41$ & 4578.908 & 65.472 & $3.11 \mathrm{e}-13$ & no \\
\hline${ }^{13} \mathrm{C}^{16} \mathrm{O}$ & $v=0-0, J=43-42$ & 4685.490 & 63.983 & $3.16 \mathrm{e}-13$ & no \\
\hline${ }^{13} \mathrm{C}^{16} \mathrm{O}$ & $v=0-0, J=44-43$ & 4791.901 & 62.562 & $3.03 \mathrm{e}-13$ & no \\
\hline${ }^{28} \mathrm{Si}^{16} \mathrm{O}$ & $v=0-0, J=11-10$ & 477.569 & 627.746 & $7.26 \mathrm{e}-14$ & no \\
\hline${ }^{28} \mathrm{Si}^{16} \mathrm{O}$ & $v=0-0, J=12-11$ & 520.740 & 575.705 & $9.52 \mathrm{e}-14$ & no \\
\hline${ }^{28} \mathrm{Si}^{16} \mathrm{O}$ & $v=0-0, J=13-12$ & 564.209 & 531.350 & $1.19 \mathrm{e}-13$ & no \\
\hline${ }^{28} \mathrm{Si}^{16} \mathrm{O}$ & $v=0-0, J=14-13$ & 607.679 & 493.340 & $1.41 \mathrm{e}-13$ & no \\
\hline${ }^{28} \mathrm{Si}^{16} \mathrm{O}$ & $v=0-0, J=15-14$ & 650.850 & 460.617 & $1.62 \mathrm{e}-13$ & no \\
\hline${ }^{28} \mathrm{Si}^{16} \mathrm{O}$ & $v=0-0, J=16-15$ & 694.319 & 431.779 & $1.81 \mathrm{e}-13$ & no \\
\hline${ }^{28} \mathrm{Si}^{16} \mathrm{O}$ & $v=0-0, J=17-16$ & 737.490 & 406.504 & $1.94 \mathrm{e}-13$ & no \\
\hline${ }^{28} \mathrm{Si}^{16} \mathrm{O}$ & $v=0-0, J=18-17$ & 780.959 & 383.877 & $2.11 \mathrm{e}-13$ & no \\
\hline${ }^{28} \mathrm{Si}^{16} \mathrm{O}$ & $v=0-0, J=19-18$ & 824.130 & 363.769 & $2.16 \mathrm{e}-13$ & no \\
\hline${ }^{28} \mathrm{Si}^{16} \mathrm{O}$ & $v=0-0, J=20-19$ & 867.600 & 345.542 & $2.29 \mathrm{e}-13$ & no \\
\hline${ }^{28} \mathrm{Si}^{16} \mathrm{O}$ & $v=0-0, J=21-20$ & 910.770 & 329.164 & $2.33 e-13$ & no \\
\hline${ }^{28} \mathrm{Si}^{16} \mathrm{O}$ & $v=0-0, J=22-21$ & 953.940 & 314.268 & $2.46 \mathrm{e}-13$ & no \\
\hline${ }^{28} \mathrm{Si}^{16} \mathrm{O}$ & $v=0-0, J=23-22$ & 997.410 & 300.571 & $2.49 \mathrm{e}-13$ & no \\
\hline${ }^{28} \mathrm{Si}^{16} \mathrm{O}$ & $v=0-0, J=24-23$ & 1040.580 & 288.101 & $2.57 e-13$ & no \\
\hline${ }^{28} \mathrm{Si}^{16} \mathrm{O}$ & $v=0-0, J=25-24$ & 1083.750 & 276.625 & $2.57 \mathrm{e}-13$ & no \\
\hline${ }^{28} \mathrm{Si}^{16} \mathrm{O}$ & $v=0-0, J=26-25$ & 1126.920 & 266.028 & $2.55 \mathrm{e}-13$ & no \\
\hline${ }^{28} \mathrm{Si}^{16} \mathrm{O}$ & $v=0-0, J=27-26$ & 1170.090 & 256.213 & $2.64 \mathrm{e}-13$ & no \\
\hline${ }^{28} \mathrm{Si}^{16} \mathrm{O}$ & $v=0-0, J=28-27$ & 1213.260 & 247.097 & $2.66 \mathrm{e}-13$ & yes \\
\hline${ }^{28} \mathrm{Si}^{16} \mathrm{O}$ & $v=0-0, J=29-28$ & 1256.430 & 238.606 & $2.60 \mathrm{e}-13$ & no \\
\hline${ }^{28} \mathrm{Si}^{16} \mathrm{O}$ & $v=0-0, J=30-29$ & 1299.601 & 230.680 & $2.66 \mathrm{e}-13$ & yes \\
\hline${ }^{28} \mathrm{Si}^{16} \mathrm{O}$ & $v=0-0, J=31-30$ & 1342.471 & 223.314 & $2.62 \mathrm{e}-13$ & no \\
\hline${ }^{28} \mathrm{Si}^{16} \mathrm{O}$ & $v=0-0, J=32-31$ & 1385.641 & 216.357 & $7.17 \mathrm{e}-14$ & yes \\
\hline${ }^{28} \mathrm{Si}^{16} \mathrm{O}$ & $v=0-0, J=33-32$ & 1428.811 & 209.820 & $2.73 \mathrm{e}-13$ & no \\
\hline${ }^{28} \mathrm{Si}^{16} \mathrm{O}$ & $v=0-0, J=34-33$ & 1471.681 & 203.707 & $9.86 \mathrm{e}-14$ & no \\
\hline
\end{tabular}


A\&A 518, L143 (2010)

Table A.1. continued.

\begin{tabular}{|c|c|c|c|c|c|}
\hline Molecule & Transition & $\begin{array}{l}\text { Frequency } \\
{[\mathrm{GHz}]}\end{array}$ & $\begin{array}{l}\text { Wavelength } \\
{[\mu \mathrm{m}]}\end{array}$ & $\begin{array}{l}\text { Integrated Flux } \\
{\left[\mathrm{erg} / \mathrm{cm}^{2} / \mathrm{s}\right]}\end{array}$ & Blend \\
\hline${ }^{28} \mathrm{Si}^{16} \mathrm{O}$ & $v=0-0, J=35-34$ & 1514.852 & 197.902 & $2.75 \mathrm{e}-13$ & yes \\
\hline${ }^{28} \mathrm{Si}^{16} \mathrm{O}$ & $v=0-0, J=36-35$ & 1557.722 & 192.456 & $1.22 \mathrm{e}-13$ & no \\
\hline${ }^{28} \mathrm{Si}^{16} \mathrm{O}$ & $v=0-0, J=37-36$ & 1600.592 & 187.301 & $2.75 \mathrm{e}-13$ & yes \\
\hline${ }^{28} \mathrm{Si}^{16} \mathrm{O}$ & $v=0-0, J=38-37$ & 1643.762 & 182.382 & $2.70 \mathrm{e}-13$ & no \\
\hline${ }^{28} \mathrm{Si}^{16} \mathrm{O}$ & $v=0-0, J=39-38$ & 1686.633 & 177.746 & $2.73 e-13$ & yes \\
\hline${ }^{28} \mathrm{Si}^{16} \mathrm{O}$ & $v=0-0, J=40-39$ & 1729.503 & 173.340 & $2.77 \mathrm{e}-13$ & no \\
\hline${ }^{28} \mathrm{Si}^{16} \mathrm{O}$ & $v=0-0, J=41-40$ & 1772.373 & 169.147 & $2.75 \mathrm{e}-13$ & no \\
\hline${ }^{28} \mathrm{Si}^{16} \mathrm{O}$ & $v=0-0, J=42-41$ & 1814.944 & 165.180 & $2.79 \mathrm{e}-13$ & no \\
\hline${ }^{28} \mathrm{Si}^{16} \mathrm{O}$ & $v=0-0, J=43-42$ & 1857.814 & 161.368 & $2.78 \mathrm{e}-13$ & yes \\
\hline${ }^{28} \mathrm{Si}^{16} \mathrm{O}$ & $v=0-0, J=44-43$ & 1900.684 & 157.729 & $2.77 \mathrm{e}-13$ & yes \\
\hline${ }^{28} \mathrm{Si}^{16} \mathrm{O}$ & $v=0-0, J=45-44$ & 1943.255 & 154.273 & $2.69 \mathrm{e}-13$ & no \\
\hline${ }^{28} \mathrm{Si}^{16} \mathrm{O}$ & $v=0-0, J=46-45$ & 1986.125 & 150.943 & $2.68 \mathrm{e}-13$ & no \\
\hline${ }^{28} \mathrm{Si}^{16} \mathrm{O}$ & $v=0-0, J=47-46$ & 2028.696 & 147.776 & $2.70 \mathrm{e}-13$ & no \\
\hline${ }^{28} \mathrm{Si}^{16} \mathrm{O}$ & $v=0-0, J=48-47$ & 2071.266 & 144.739 & $2.73 e-13$ & yes \\
\hline${ }^{28} \mathrm{Si}^{16} \mathrm{O}$ & $v=0-0, J=49-48$ & 2113.837 & 141.824 & $2.64 \mathrm{e}-13$ & no \\
\hline${ }^{28} \mathrm{Si}^{16} \mathrm{O}$ & $v=0-0, J=50-49$ & 2156.408 & 139.024 & $2.69 \mathrm{e}-13$ & no \\
\hline${ }^{28} \mathrm{Si}^{16} \mathrm{O}$ & $v=0-0, J=51-50$ & 2198.978 & 136.333 & $2.60 \mathrm{e}-13$ & yes \\
\hline${ }^{28} \mathrm{Si}^{16} \mathrm{O}$ & $v=0-0, J=52-51$ & 2241.549 & 133.743 & $2.62 \mathrm{e}-13$ & no \\
\hline${ }^{28} \mathrm{Si}^{16} \mathrm{O}$ & $v=0-0, J=53-52$ & 2283.819 & 131.268 & $2.59 \mathrm{e}-13$ & yes \\
\hline${ }^{28} \mathrm{Si}^{16} \mathrm{O}$ & $v=0-0, J=54-53$ & 2326.390 & 128.866 & $2.48 \mathrm{e}-13$ & yes \\
\hline${ }^{28} \mathrm{Si}^{16} \mathrm{O}$ & $v=0-0, J=55-54$ & 2368.661 & 126.566 & $2.51 \mathrm{e}-13$ & yes \\
\hline${ }^{28} \mathrm{Si}^{16} \mathrm{O}$ & $v=0-0, J=56-55$ & 2410.931 & 124.347 & $2.48 \mathrm{e}-13$ & yes \\
\hline${ }^{28} \mathrm{Si}^{16} \mathrm{O}$ & $v=0-0, J=57-56$ & 2453.202 & 122.205 & $2.43 \mathrm{e}-13$ & no \\
\hline${ }^{28} \mathrm{Si}^{16} \mathrm{O}$ & $v=0-0, J=58-57$ & 2495.473 & 120.135 & $2.37 e-13$ & yes \\
\hline${ }^{28} \mathrm{Si}^{16} \mathrm{O}$ & $v=0-0, J=59-58$ & 2537.744 & 118.133 & $2.27 \mathrm{e}-13$ & no \\
\hline${ }^{28} \mathrm{Si}^{16} \mathrm{O}$ & $v=0-0, J=60-59$ & 2580.014 & 116.198 & $2.27 \mathrm{e}-13$ & yes \\
\hline${ }^{28} \mathrm{Si}^{16} \mathrm{O}$ & $v=0-0, J=61-60$ & 2621.985 & 114.338 & $2.23 \mathrm{e}-13$ & yes \\
\hline${ }^{28} \mathrm{Si}^{16} \mathrm{O}$ & $v=0-0, J=62-61$ & 2664.256 & 112.524 & $2.17 \mathrm{e}-13$ & yes \\
\hline${ }^{28} \mathrm{Si}^{16} \mathrm{O}$ & $v=0-0, J=63-62$ & 2706.227 & 110.779 & $2.10 \mathrm{e}-13$ & no \\
\hline${ }^{28} \mathrm{Si}^{16} \mathrm{O}$ & $v=0-0, J=64-63$ & 2748.198 & 109.087 & $2.05 e-13$ & yes \\
\hline${ }^{28} \mathrm{Si}^{16} \mathrm{O}$ & $v=0-0, J=65-64$ & 2790.169 & 107.446 & $2.01 \mathrm{e}-13$ & no \\
\hline${ }^{28} \mathrm{Si}^{16} \mathrm{O}$ & $v=0-0, J=66-65$ & 2832.140 & 105.854 & $1.94 \mathrm{e}-13$ & yes \\
\hline${ }^{28} \mathrm{Si}^{16} \mathrm{O}$ & $v=0-0, J=67-66$ & 2874.111 & 104.308 & $1.84 \mathrm{e}-13$ & yes \\
\hline${ }^{28} \mathrm{Si}^{16} \mathrm{O}$ & $v=0-0, J=68-67$ & 2915.782 & 102.817 & $1.80 \mathrm{e}-13$ & yes \\
\hline${ }^{28} \mathrm{Si}^{16} \mathrm{O}$ & $v=0-0, J=72-71$ & 3082.467 & 97.257 & $1.56 \mathrm{e}-13$ & no \\
\hline${ }^{28} \mathrm{Si}^{16} \mathrm{O}$ & $v=0-0, J=73-72$ & 3124.138 & 95.960 & $1.52 \mathrm{e}-13$ & yes \\
\hline${ }^{28} \mathrm{Si}^{16} \mathrm{O}$ & $v=0-0, J=74-73$ & 3165.809 & 94.697 & $1.46 \mathrm{e}-13$ & yes \\
\hline${ }^{28} \mathrm{Si}^{16} \mathrm{O}$ & $v=0-0, J=75-74$ & 3207.180 & 93.475 & $1.37 \mathrm{e}-13$ & yes \\
\hline${ }^{28} \mathrm{Si}^{16} \mathrm{O}$ & $v=0-0, J=76-75$ & 3248.552 & 92.285 & $1.34 \mathrm{e}-13$ & no \\
\hline${ }^{28} \mathrm{Si}^{16} \mathrm{O}$ & $v=0-0, J=77-76$ & 3289.923 & 91.124 & $1.28 \mathrm{e}-13$ & yes \\
\hline${ }^{28} \mathrm{Si}^{16} \mathrm{O}$ & $v=0-0, J=78-77$ & 3331.294 & 89.993 & $1.19 \mathrm{e}-13$ & yes \\
\hline${ }^{28} \mathrm{Si}^{16} \mathrm{O}$ & $v=0-0, J=79-78$ & 3372.666 & 88.889 & $1.17 \mathrm{e}-13$ & no \\
\hline${ }^{28} \mathrm{Si}^{16} \mathrm{O}$ & $v=0-0, J=80-79$ & 3413.737 & 87.819 & $1.13 \mathrm{e}-13$ & yes \\
\hline${ }^{28} \mathrm{Si}^{16} \mathrm{O}$ & $v=0-0, J=81-80$ & 3454.809 & 86.775 & $1.08 \mathrm{e}-13$ & yes \\
\hline${ }^{28} \mathrm{Si}^{16} \mathrm{O}$ & $v=0-0, J=82-81$ & 3496.180 & 85.749 & $1.03 \mathrm{e}-13$ & yes \\
\hline${ }^{28} \mathrm{Si}^{16} \mathrm{O}$ & $v=0-0, J=83-82$ & 3536.952 & 84.760 & $9.76 \mathrm{e}-14$ & yes \\
\hline${ }^{28} \mathrm{Si}^{16} \mathrm{O}$ & $v=0-0, J=84-83$ & 3578.024 & 83.787 & $9.20 \mathrm{e}-14$ & yes \\
\hline${ }^{28} \mathrm{Si}^{16} \mathrm{O}$ & $v=0-0, J=85-84$ & 3619.095 & 82.836 & $8.84 \mathrm{e}-14$ & yes \\
\hline${ }^{28} \mathrm{Si}^{16} \mathrm{O}$ & $v=0-0, J=86-85$ & 3659.867 & 81.913 & $8.23 e-14$ & yes \\
\hline${ }^{28} \mathrm{Si}^{16} \mathrm{O}$ & $v=0-0, J=87-86$ & 3700.639 & 81.011 & $8.11 \mathrm{e}-14$ & yes \\
\hline${ }^{28} \mathrm{Si}^{16} \mathrm{O}$ & $v=0-0, J=88-87$ & 3741.410 & 80.128 & $7.74 \mathrm{e}-14$ & yes \\
\hline${ }^{28} \mathrm{Si}^{16} \mathrm{O}$ & $v=0-0, J=89-88$ & 3782.182 & 79.264 & $7.36 \mathrm{e}-14$ & yes \\
\hline${ }^{28} \mathrm{Si}^{16} \mathrm{O}$ & $v=0-0, J=90-89$ & 3822.654 & 78.425 & $7.13 \mathrm{e}-14$ & yes \\
\hline${ }^{28} \mathrm{Si}^{16} \mathrm{O}$ & $v=1-1, J=39-38$ & 1674.641 & 179.019 & $4.06 \mathrm{e}-14$ & yes \\
\hline${ }^{28} \mathrm{Si}^{16} \mathrm{O}$ & $v=1-1, J=40-39$ & 1717.211 & 174.581 & $4.28 \mathrm{e}-14$ & yes \\
\hline${ }^{28} \mathrm{Si}^{16} \mathrm{O}$ & $v=1-1, J=41-40$ & 1759.782 & 170.358 & $4.40 \mathrm{e}-14$ & yes \\
\hline
\end{tabular}


Table A.1. continued.

\begin{tabular}{|c|c|c|c|c|c|}
\hline Molecule & Transition & $\begin{array}{l}\text { Frequency } \\
{[\mathrm{GHz}]}\end{array}$ & $\begin{array}{l}\text { Wavelength } \\
{[\mu \mathrm{m}]}\end{array}$ & $\begin{array}{l}\text { Integrated Flux } \\
{\left[\mathrm{erg} / \mathrm{cm}^{2} / \mathrm{s}\right]}\end{array}$ & Blend \\
\hline${ }^{28} \mathrm{Si}^{16} \mathrm{O}$ & $v=1-1, J=42-41$ & 1802.353 & 166.334 & $4.74 \mathrm{e}-14$ & no \\
\hline${ }^{28} \mathrm{Si}^{16} \mathrm{O}$ & $v=1-1, J=43-42$ & 1844.923 & 162.496 & $4.88 \mathrm{e}-14$ & yes \\
\hline${ }^{28} \mathrm{Si}^{16} \mathrm{O}$ & $v=1-1, J=44-43$ & 1887.194 & 158.856 & $5.15 e-14$ & yes \\
\hline${ }^{28} \mathrm{Si}^{16} \mathrm{O}$ & $v=1-1, J=45-44$ & 1929.764 & 155.352 & $5.28 \mathrm{e}-14$ & no \\
\hline${ }^{28} \mathrm{Si}^{16} \mathrm{O}$ & $v=1-1, J=46-45$ & 1972.035 & 152.022 & $5.48 \mathrm{e}-14$ & yes \\
\hline${ }^{28} \mathrm{Si}^{16} \mathrm{O}$ & $v=1-1, J=47-46$ & 2014.606 & 148.809 & $5.53 \mathrm{e}-14$ & yes \\
\hline${ }^{28} \mathrm{Si}^{16} \mathrm{O}$ & $v=1-1, J=48-47$ & 2056.876 & 145.751 & $5.64 \mathrm{e}-14$ & yes \\
\hline${ }^{28} \mathrm{Si}^{16} \mathrm{O}$ & $v=1-1, J=49-48$ & 2099.147 & 142.816 & $5.82 \mathrm{e}-14$ & no \\
\hline${ }^{28} \mathrm{Si}^{32} \mathrm{~S}$ & $v=0-0, J=26-25$ & 471.608 & 635.682 & $1.29 \mathrm{e}-13$ & yes \\
\hline${ }^{28} \mathrm{Si}^{32} \mathrm{~S}$ & $v=0-0, J=27-26$ & 489.713 & 612.180 & $1.45 \mathrm{e}-13$ & yes \\
\hline${ }^{28} \mathrm{Si}^{32} \mathrm{~S}$ & $v=0-0, J=28-27$ & 507.813 & 590.360 & $1.47 \mathrm{e}-13$ & no \\
\hline${ }^{28} \mathrm{Si}^{32} \mathrm{~S}$ & $v=0-0, J=29-28$ & 525.910 & 570.045 & $1.61 \mathrm{e}-13$ & no \\
\hline${ }^{28} \mathrm{Si}^{32} \mathrm{~S}$ & $v=0-0, J=30-29$ & 544.003 & 551.087 & $1.61 \mathrm{e}-13$ & no \\
\hline${ }^{28} \mathrm{Si}^{32} \mathrm{~S}$ & $v=0-0, J=31-30$ & 562.091 & 533.352 & $1.76 \mathrm{e}-13$ & no \\
\hline${ }^{28} \mathrm{Si}^{32} \mathrm{~S}$ & $v=0-0, J=32-31$ & 580.175 & 516.728 & $1.74 \mathrm{e}-13$ & no \\
\hline${ }^{28} \mathrm{Si}^{32} \mathrm{~S}$ & $v=0-0, J=33-32$ & 598.254 & 501.112 & $1.85 \mathrm{e}-13$ & no \\
\hline${ }^{28} \mathrm{Si}^{32} \mathrm{~S}$ & $v=0-0, J=34-33$ & 616.328 & 486.417 & $1.84 \mathrm{e}-13$ & no \\
\hline${ }^{28} \mathrm{Si}^{32} \mathrm{~S}$ & $v=0-0, J=35-34$ & 634.398 & 472.562 & $1.91 \mathrm{e}-13$ & no \\
\hline${ }^{28} \mathrm{Si}^{32} \mathrm{~S}$ & $v=0-0, J=36-35$ & 652.463 & 459.478 & $1.93 \mathrm{e}-13$ & no \\
\hline${ }^{28} \mathrm{Si}^{32} \mathrm{~S}$ & $v=0-0, J=37-36$ & 670.522 & 447.103 & $1.93 \mathrm{e}-13$ & no \\
\hline${ }^{28} \mathrm{Si}^{32} \mathrm{~S}$ & $v=0-0, J=38-37$ & 688.576 & 435.380 & $1.98 \mathrm{e}-13$ & no \\
\hline${ }^{28} \mathrm{Si}^{32} \mathrm{~S}$ & $v=0-0, J=39-38$ & 706.625 & 424.260 & $1.93 \mathrm{e}-13$ & yes \\
\hline${ }^{28} \mathrm{Si}^{32} \mathrm{~S}$ & $v=0-0, J=40-39$ & 724.668 & 413.696 & $2.00 \mathrm{e}-13$ & no \\
\hline${ }^{28} \mathrm{Si}^{32} \mathrm{~S}$ & $v=0-0, J=41-40$ & 742.705 & 403.649 & $2.00 \mathrm{e}-13$ & no \\
\hline${ }^{28} \mathrm{Si}^{32} \mathrm{~S}$ & $v=0-0, J=42-41$ & 760.737 & 394.082 & $1.97 \mathrm{e}-13$ & no \\
\hline${ }^{28} \mathrm{Si}^{32} \mathrm{~S}$ & $v=0-0, J=43-42$ & 778.762 & 384.960 & $2.03 \mathrm{e}-13$ & no \\
\hline${ }^{28} \mathrm{Si}^{32} \mathrm{~S}$ & $v=0-0, J=44-43$ & 796.782 & 376.254 & $2.00 \mathrm{e}-13$ & yes \\
\hline${ }^{28} \mathrm{Si}^{32} \mathrm{~S}$ & $v=0-0, J=45-44$ & 814.795 & 367.936 & $1.99 \mathrm{e}-13$ & no \\
\hline${ }^{28} \mathrm{Si}^{32} \mathrm{~S}$ & $v=0-0, J=46-45$ & 832.801 & 359.981 & $2.04 \mathrm{e}-13$ & yes \\
\hline${ }^{28} \mathrm{Si}^{32} \mathrm{~S}$ & $v=0-0, J=47-46$ & 850.801 & 352.365 & $2.02 \mathrm{e}-13$ & no \\
\hline${ }^{28} \mathrm{Si}^{32} \mathrm{~S}$ & $v=0-0, J=48-47$ & 868.795 & 345.067 & $1.98 \mathrm{e}-13$ & no \\
\hline${ }^{28} \mathrm{Si}^{32} \mathrm{~S}$ & $v=0-0, J=49-48$ & 886.781 & 338.068 & $2.04 \mathrm{e}-13$ & yes \\
\hline${ }^{28} \mathrm{Si}^{32} \mathrm{~S}$ & $v=0-0, J=50-49$ & 904.760 & 331.350 & $2.05 \mathrm{e}-13$ & no \\
\hline${ }^{28} \mathrm{Si}^{32} \mathrm{~S}$ & $v=0-0, J=51-50$ & 922.733 & 324.896 & $2.00 \mathrm{e}-13$ & yes \\
\hline${ }^{28} \mathrm{Si}^{32} \mathrm{~S}$ & $v=0-0, J=52-51$ & 940.697 & 318.692 & $2.01 \mathrm{e}-13$ & no \\
\hline${ }^{28} \mathrm{Si}^{32} \mathrm{~S}$ & $v=0-0, J=53-52$ & 958.655 & 312.722 & $2.06 \mathrm{e}-13$ & no \\
\hline${ }^{28} \mathrm{Si}^{32} \mathrm{~S}$ & $v=0-0, J=54-53$ & 976.605 & 306.974 & $2.07 \mathrm{e}-13$ & yes \\
\hline${ }^{28} \mathrm{Si}^{32} \mathrm{~S}$ & $v=0-0, J=55-54$ & 994.547 & 301.436 & $2.05 \mathrm{e}-13$ & no \\
\hline${ }^{28} \mathrm{Si}^{32} \mathrm{~S}$ & $v=0-0, J=56-55$ & 1012.481 & 296.097 & $2.01 \mathrm{e}-13$ & no \\
\hline${ }^{28} \mathrm{Si}^{32} \mathrm{~S}$ & $v=0-0, J=57-56$ & 1030.407 & 290.946 & $2.03 \mathrm{e}-13$ & no \\
\hline${ }^{28} \mathrm{Si}^{32} \mathrm{~S}$ & $v=0-0, J=58-57$ & 1048.325 & 285.973 & $2.07 \mathrm{e}-13$ & no \\
\hline${ }^{28} \mathrm{Si}^{32} \mathrm{~S}$ & $v=0-0, J=59-58$ & 1066.235 & 281.169 & $2.09 \mathrm{e}-13$ & yes \\
\hline${ }^{28} \mathrm{Si}^{32} \mathrm{~S}$ & $v=0-0, J=60-59$ & 1084.136 & 276.527 & $2.09 \mathrm{e}-13$ & no \\
\hline${ }^{28} \mathrm{Si}^{32} \mathrm{~S}$ & $v=0-0, J=61-60$ & 1102.029 & 272.037 & $2.09 \mathrm{e}-13$ & yes \\
\hline${ }^{28} \mathrm{Si}^{32} \mathrm{~S}$ & $v=0-0, J=62-61$ & 1119.913 & 267.693 & $2.08 \mathrm{e}-13$ & yes \\
\hline${ }^{28} \mathrm{Si}^{32} \mathrm{~S}$ & $v=0-0, J=63-62$ & 1137.788 & 263.487 & $2.07 \mathrm{e}-13$ & no \\
\hline${ }^{28} \mathrm{Si}^{32} \mathrm{~S}$ & $v=0-0, J=64-63$ & 1155.654 & 259.414 & $2.06 \mathrm{e}-13$ & yes \\
\hline${ }^{28} \mathrm{Si}^{32} \mathrm{~S}$ & $v=0-0, J=65-64$ & 1173.511 & 255.466 & $2.05 \mathrm{e}-13$ & yes \\
\hline${ }^{28} \mathrm{Si}^{32} \mathrm{~S}$ & $v=0-0, J=66-65$ & 1191.358 & 251.639 & $2.04 \mathrm{e}-13$ & no \\
\hline${ }^{28} \mathrm{Si}^{32} \mathrm{~S}$ & $v=0-0, J=67-66$ & 1209.196 & 247.927 & $2.04 \mathrm{e}-13$ & yes \\
\hline${ }^{28} \mathrm{Si}^{32} \mathrm{~S}$ & $v=0-0, J=68-67$ & 1227.025 & 244.325 & $2.04 \mathrm{e}-13$ & no \\
\hline${ }^{28} \mathrm{Si}^{32} \mathrm{~S}$ & $v=0-0, J=69-68$ & 1244.843 & 240.827 & $2.04 \mathrm{e}-13$ & yes \\
\hline${ }^{28} \mathrm{Si}^{32} \mathrm{~S}$ & $v=0-0, J=70-69$ & 1262.652 & 237.431 & $2.05 \mathrm{e}-13$ & no \\
\hline${ }^{28} \mathrm{Si}^{32} \mathrm{~S}$ & $v=0-0, J=71-70$ & 1280.451 & 234.130 & $2.05 \mathrm{e}-13$ & no \\
\hline${ }^{28} \mathrm{Si}^{32} \mathrm{~S}$ & $v=0-0, J=72-71$ & 1298.240 & 230.922 & $2.06 \mathrm{e}-13$ & yes \\
\hline${ }^{28} \mathrm{Si}^{32} \mathrm{~S}$ & $v=0-0, J=73-72$ & 1316.018 & 227.803 & $2.05 \mathrm{e}-13$ & no \\
\hline
\end{tabular}


A\&A 518, L143 (2010)

Table A.1. continued.

\begin{tabular}{|c|c|c|c|c|c|}
\hline Molecule & Transition & $\begin{array}{l}\text { Frequency } \\
{[\mathrm{GHz}]}\end{array}$ & $\begin{array}{l}\text { Wavelength } \\
{[\mu \mathrm{m}]}\end{array}$ & $\begin{array}{l}\text { Integrated Flux } \\
{\left[\mathrm{erg} / \mathrm{cm}^{2} / \mathrm{s}\right]}\end{array}$ & Blend \\
\hline${ }^{28} \mathrm{Si}^{32} \mathrm{~S}$ & $v=0-0, J=74-73$ & 1333.786 & 224.768 & $2.04 \mathrm{e}-13$ & yes \\
\hline${ }^{28} \mathrm{Si}^{32} \mathrm{~S}$ & $v=0-0, J=75-74$ & 1351.543 & 221.815 & $2.00 \mathrm{e}-13$ & no \\
\hline${ }^{28} \mathrm{Si}^{32} \mathrm{~S}$ & $v=0-0, J=76-75$ & 1369.290 & 218.940 & $4.81 \mathrm{e}-14$ & yes \\
\hline${ }^{28} \mathrm{Si}^{32} \mathrm{~S}$ & $v=0-0, J=77-76$ & 1387.025 & 216.141 & $1.96 \mathrm{e}-13$ & yes \\
\hline${ }^{28} \mathrm{Si}^{32} \mathrm{~S}$ & $v=0-0, J=78-77$ & 1404.750 & 213.413 & $5.78 \mathrm{e}-14$ & no \\
\hline${ }^{28} \mathrm{Si}^{32} \mathrm{~S}$ & $v=0-0, J=79-78$ & 1422.463 & 210.756 & $1.97 \mathrm{e}-13$ & yes \\
\hline${ }^{28} \mathrm{Si}^{32} \mathrm{~S}$ & $v=0-0, J=80-79$ & 1440.165 & 208.165 & $6.35 \mathrm{e}-14$ & no \\
\hline${ }^{28} \mathrm{Si}^{32} \mathrm{~S}$ & $v=0-0, J=81-80$ & 1457.856 & 205.639 & $6.63 \mathrm{e}-14$ & no \\
\hline${ }^{28} \mathrm{Si}^{32} \mathrm{~S}$ & $v=0-0, J=82-81$ & 1475.535 & 203.175 & $7.07 \mathrm{e}-14$ & no \\
\hline${ }^{28} \mathrm{Si}^{32} \mathrm{~S}$ & $v=0-0, J=83-82$ & 1493.202 & 200.772 & $7.24 \mathrm{e}-14$ & yes \\
\hline${ }^{28} \mathrm{Si}^{32} \mathrm{~S}$ & $v=0-0, J=84-83$ & 1510.858 & 198.425 & $1.83 \mathrm{e}-13$ & yes \\
\hline${ }^{28} \mathrm{Si}^{32} \mathrm{~S}$ & $v=0-0, J=85-84$ & 1528.501 & 196.135 & $7.80 \mathrm{e}-14$ & no \\
\hline${ }^{28} \mathrm{Si}^{32} \mathrm{~S}$ & $v=0-0, J=86-85$ & 1546.132 & 193.898 & $1.79 \mathrm{e}-13$ & no \\
\hline${ }^{28} \mathrm{Si}^{32} \mathrm{~S}$ & $v=0-0, J=87-86$ & 1563.751 & 191.714 & $8.13 \mathrm{e}-14$ & no \\
\hline${ }^{28} \mathrm{Si}^{32} \mathrm{~S}$ & $v=0-0, J=88-87$ & 1581.357 & 189.579 & $1.78 \mathrm{e}-13$ & yes \\
\hline${ }^{28} \mathrm{Si}^{32} \mathrm{~S}$ & $v=0-0, J=89-88$ & 1598.951 & 187.493 & $1.71 \mathrm{e}-13$ & yes \\
\hline${ }^{28} \mathrm{Si}^{32} \mathrm{~S}$ & $v=0-0, J=90-89$ & 1616.532 & 185.454 & $1.72 \mathrm{e}-13$ & no \\
\hline${ }^{28} \mathrm{Si}^{32} \mathrm{~S}$ & $v=0-0, J=91-90$ & 1634.100 & 183.460 & $1.72 \mathrm{e}-13$ & no \\
\hline${ }^{28} \mathrm{Si}^{32} \mathrm{~S}$ & $v=0-0, J=92-91$ & 1651.655 & 181.510 & $1.66 \mathrm{e}-13$ & yes \\
\hline${ }^{28} \mathrm{Si}^{32} \mathrm{~S}$ & $v=0-0, J=93-92$ & 1669.197 & 179.603 & $1.65 \mathrm{e}-13$ & yes \\
\hline${ }^{28} \mathrm{Si}^{32} \mathrm{~S}$ & $v=0-0, J=94-93$ & 1686.725 & 177.736 & $1.60 \mathrm{e}-13$ & yes \\
\hline${ }^{28} \mathrm{Si}^{32} \mathrm{~S}$ & $v=0-0, J=95-94$ & 1704.240 & 175.910 & $1.60 \mathrm{e}-13$ & no \\
\hline${ }^{28} \mathrm{Si}^{32} \mathrm{~S}$ & $v=0-0, J=96-95$ & 1721.742 & 174.122 & $1.55 \mathrm{e}-13$ & no \\
\hline${ }^{28} \mathrm{Si}^{32} \mathrm{~S}$ & $v=0-0, J=97-96$ & 1739.229 & 172.371 & $1.55 \mathrm{e}-13$ & no \\
\hline${ }^{28} \mathrm{Si}^{32} \mathrm{~S}$ & $v=0-0, J=98-97$ & 1756.703 & 170.656 & $1.53 \mathrm{e}-13$ & yes \\
\hline${ }^{28} \mathrm{Si}^{32} \mathrm{~S}$ & $v=0-0, J=99-98$ & 1774.163 & 168.977 & $1.48 \mathrm{e}-13$ & yes \\
\hline${ }^{28} \mathrm{Si}^{32} \mathrm{~S}$ & $v=0-0, J=100-99$ & 1791.608 & 167.331 & $1.44 \mathrm{e}-13$ & yes \\
\hline${ }^{28} \mathrm{Si}^{32} \mathrm{~S}$ & $v=0-0, J=101-100$ & 1809.039 & 165.719 & $1.42 \mathrm{e}-13$ & yes \\
\hline${ }^{28} \mathrm{Si}^{32} \mathrm{~S}$ & $v=0-0, J=102-101$ & 1826.456 & 164.139 & $1.41 \mathrm{e}-13$ & no \\
\hline${ }^{28} \mathrm{Si}^{32} \mathrm{~S}$ & $v=0-0, J=103-102$ & 1843.858 & 162.590 & $1.36 \mathrm{e}-13$ & yes \\
\hline${ }^{28} \mathrm{Si}^{32} \mathrm{~S}$ & $v=0-0, J=104-103$ & 1861.245 & 161.071 & $1.31 \mathrm{e}-13$ & no \\
\hline${ }^{28} \mathrm{Si}^{32} \mathrm{~S}$ & $v=0-0, J=105-104$ & 1878.618 & 159.581 & $1.32 \mathrm{e}-13$ & no \\
\hline${ }^{28} \mathrm{Si}^{32} \mathrm{~S}$ & $v=0-0, J=106-105$ & 1895.975 & 158.120 & $1.27 \mathrm{e}-13$ & yes \\
\hline${ }^{28} \mathrm{Si}^{32} \mathrm{~S}$ & $v=0-0, J=107-106$ & 1913.318 & 156.687 & $1.26 \mathrm{e}-13$ & no \\
\hline${ }^{28} \mathrm{Si}^{32} \mathrm{~S}$ & $v=0-0, J=108-107$ & 1930.644 & 155.281 & $1.19 \mathrm{e}-13$ & yes \\
\hline${ }^{28} \mathrm{Si}^{32} \mathrm{~S}$ & $v=0-0, J=109-108$ & 1947.956 & 153.901 & $1.17 \mathrm{e}-13$ & yes \\
\hline${ }^{28} \mathrm{Si}^{32} \mathrm{~S}$ & $v=0-0, J=110-109$ & 1965.251 & 152.547 & $1.13 \mathrm{e}-13$ & no \\
\hline${ }^{28} \mathrm{Si}^{32} \mathrm{~S}$ & $v=0-0, J=111-110$ & 1982.531 & 151.217 & $1.15 \mathrm{e}-13$ & yes \\
\hline${ }^{28} \mathrm{Si}^{32} \mathrm{~S}$ & $v=0-0, J=112-111$ & 1999.795 & 149.912 & $1.08 \mathrm{e}-13$ & yes \\
\hline${ }^{28} \mathrm{Si}^{32} \mathrm{~S}$ & $v=0-0, J=113-112$ & 2017.043 & 148.630 & $1.06 \mathrm{e}-13$ & no \\
\hline${ }^{28} \mathrm{Si}^{32} \mathrm{~S}$ & $v=0-0, J=114-113$ & 2034.275 & 147.371 & $1.03 \mathrm{e}-13$ & yes \\
\hline${ }^{28} \mathrm{Si}^{32} \mathrm{~S}$ & $v=0-0, J=115-114$ & 2051.491 & 146.134 & $1.03 \mathrm{e}-13$ & no \\
\hline${ }^{28} \mathrm{Si}^{32} \mathrm{~S}$ & $v=0-0, J=116-115$ & 2068.690 & 144.919 & $1.00 \mathrm{e}-13$ & yes \\
\hline${ }^{28} \mathrm{Si}^{32} \mathrm{~S}$ & $v=0-0, J=117-116$ & 2085.872 & 143.725 & $9.45 \mathrm{e}-14$ & no \\
\hline${ }^{28} \mathrm{Si}^{32} \mathrm{~S}$ & $v=0-0, J=118-117$ & 2103.037 & 142.552 & $9.44 \mathrm{e}-14$ & no \\
\hline${ }^{28} \mathrm{Si}^{32} \mathrm{~S}$ & $v=0-0, J=119-118$ & 2120.186 & 141.399 & $9.06 \mathrm{e}-14$ & yes \\
\hline${ }^{28} \mathrm{Si}^{32} \mathrm{~S}$ & $v=0-0, J=120-119$ & 2137.318 & 140.266 & $8.85 \mathrm{e}-14$ & yes \\
\hline${ }^{28} \mathrm{Si}^{32} \mathrm{~S}$ & $v=0-0, J=121-120$ & 2154.432 & 139.151 & $8.56 \mathrm{e}-14$ & yes \\
\hline${ }^{28} \mathrm{Si}^{32} \mathrm{~S}$ & $v=0-0, J=122-121$ & 2171.529 & 138.056 & $8.17 \mathrm{e}-14$ & no \\
\hline${ }^{28} \mathrm{Si}^{32} \mathrm{~S}$ & $v=0-0, J=123-122$ & 2188.609 & 136.979 & $7.89 \mathrm{e}-14$ & yes \\
\hline${ }^{28} \mathrm{Si}^{32} \mathrm{~S}$ & $v=0-0, J=124-123$ & 2205.671 & 135.919 & $7.78 \mathrm{e}-14$ & yes \\
\hline${ }^{28} \mathrm{Si}^{32} \mathrm{~S}$ & $v=1-1, J=50-49$ & 900.341 & 332.977 & $3.26 \mathrm{e}-14$ & yes \\
\hline${ }^{28} \mathrm{Si}^{32} \mathrm{~S}$ & $v=1-1, J=51-50$ & 918.224 & 326.491 & $3.48 \mathrm{e}-14$ & no \\
\hline${ }^{28} \mathrm{Si}^{32} \mathrm{~S}$ & $v=1-1, J=52-51$ & 936.101 & 320.257 & $3.69 \mathrm{e}-14$ & no \\
\hline${ }^{28} \mathrm{Si}^{32} \mathrm{~S}$ & $v=1-1, J=53-52$ & 953.970 & 314.258 & $3.89 \mathrm{e}-14$ & yes \\
\hline${ }^{28} \mathrm{Si}^{32} \mathrm{~S}$ & $v=1-1, J=54-53$ & 971.831 & 308.482 & $4.08 \mathrm{e}-14$ & yes \\
\hline
\end{tabular}


Table A.1. continued.

\begin{tabular}{|c|c|c|c|c|c|}
\hline Molecule & Transition & $\begin{array}{l}\text { Frequency } \\
{[\mathrm{GHz}]}\end{array}$ & $\begin{array}{l}\text { Wavelength } \\
{[\mu \mathrm{m}]}\end{array}$ & $\begin{array}{l}\text { Integrated Flux } \\
{\left[\mathrm{erg} / \mathrm{cm}^{2} / \mathrm{s}\right]}\end{array}$ & Blend \\
\hline${ }^{28} \mathrm{Si}^{32} \mathrm{~S}$ & $v=1-1, J=55-54$ & 989.685 & 302.917 & $4.28 \mathrm{e}-14$ & yes \\
\hline${ }^{28} \mathrm{Si}^{32} \mathrm{~S}$ & $v=1-1, J=56-55$ & 1007.530 & 297.552 & $4.47 \mathrm{e}-14$ & no \\
\hline${ }^{28} \mathrm{Si}^{32} \mathrm{~S}$ & $v=1-1, J=57-56$ & 1025.368 & 292.376 & $4.67 \mathrm{e}-14$ & yes \\
\hline${ }^{28} \mathrm{Si}^{32} \mathrm{~S}$ & $v=1-1, J=58-57$ & 1043.197 & 287.378 & $4.86 \mathrm{e}-14$ & no \\
\hline${ }^{28} \mathrm{Si}^{32} \mathrm{~S}$ & $v=1-1, J=59-58$ & 1061.018 & 282.552 & $5.03 \mathrm{e}-14$ & yes \\
\hline${ }^{28} \mathrm{Si}^{32} \mathrm{~S}$ & $v=1-1, J=60-59$ & 1078.831 & 277.886 & $5.21 \mathrm{e}-14$ & yes \\
\hline${ }^{28} \mathrm{Si}^{32} \mathrm{~S}$ & $v=1-1, J=61-60$ & 1096.635 & 273.375 & $5.37 \mathrm{e}-14$ & yes \\
\hline${ }^{28} \mathrm{Si}^{32} \mathrm{~S}$ & $v=1-1, J=62-61$ & 1114.430 & 269.010 & $5.51 \mathrm{e}-14$ & yes \\
\hline${ }^{28} \mathrm{Si}^{32} \mathrm{~S}$ & $v=1-1, J=63-62$ & 1132.217 & 264.784 & $5.61 \mathrm{e}-14$ & yes \\
\hline${ }^{28} \mathrm{Si}^{32} \mathrm{~S}$ & $v=1-1, J=64-63$ & 1149.994 & 260.690 & $5.68 \mathrm{e}-14$ & yes \\
\hline${ }^{28} \mathrm{Si}^{32} \mathrm{~S}$ & $v=1-1, J=65-64$ & 1167.763 & 256.724 & $5.84 \mathrm{e}-14$ & yes \\
\hline${ }^{28} \mathrm{Si}^{32} \mathrm{~S}$ & $v=1-1, J=66-65$ & 1185.522 & 252.878 & $6.09 \mathrm{e}-14$ & yes \\
\hline${ }^{28} \mathrm{Si}^{32} \mathrm{~S}$ & $v=1-1, J=67-66$ & 1203.271 & 249.148 & $6.29 \mathrm{e}-14$ & yes \\
\hline${ }^{28} \mathrm{Si}^{32} \mathrm{~S}$ & $v=1-1, J=68-67$ & 1221.011 & 245.528 & $6.42 \mathrm{e}-14$ & yes \\
\hline${ }^{28} \mathrm{Si}^{32} \mathrm{~S}$ & $v=1-1, J=69-68$ & 1238.741 & 242.014 & $6.46 \mathrm{e}-14$ & yes \\
\hline${ }^{28} \mathrm{Si}^{32} \mathrm{~S}$ & $v=1-1, J=70-69$ & 1256.461 & 238.601 & $6.40 \mathrm{e}-14$ & yes \\
\hline${ }^{28} \mathrm{Si}^{32} \mathrm{~S}$ & $v=1-1, J=71-70$ & 1274.171 & 235.284 & $6.70 \mathrm{e}-14$ & no \\
\hline${ }^{28} \mathrm{Si}^{32} \mathrm{~S}$ & $v=1-1, J=72-71$ & 1291.871 & 232.061 & $6.87 e-14$ & yes \\
\hline${ }^{28} \mathrm{Si}^{32} \mathrm{~S}$ & $v=1-1, J=73-72$ & 1309.561 & 228.926 & $6.86 \mathrm{e}-14$ & yes \\
\hline${ }^{28} \mathrm{Si}^{32} \mathrm{~S}$ & $v=1-1, J=74-73$ & 1327.240 & 225.877 & $6.86 \mathrm{e}-14$ & yes \\
\hline${ }^{28} \mathrm{Si}^{32} \mathrm{~S}$ & $v=1-1, J=75-74$ & 1344.908 & 222.909 & $7.11 \mathrm{e}-14$ & yes \\
\hline${ }^{28} \mathrm{Si}^{32} \mathrm{~S}$ & $v=1-1, J=76-75$ & 1362.566 & 220.021 & $1.68 \mathrm{e}-14$ & no \\
\hline${ }^{28} \mathrm{Si}^{32} \mathrm{~S}$ & $v=1-1, J=77-76$ & 1380.213 & 217.207 & $1.84 \mathrm{e}-14$ & yes \\
\hline${ }^{28} \mathrm{Si}^{32} \mathrm{~S}$ & $v=1-1, J=78-77$ & 1397.849 & 214.467 & $2.06 \mathrm{e}-14$ & yes \\
\hline${ }^{28} \mathrm{Si}^{32} \mathrm{~S}$ & $v=1-1, J=79-78$ & 1415.473 & 211.797 & $2.16 \mathrm{e}-14$ & yes \\
\hline${ }^{28} \mathrm{Si}^{32} \mathrm{~S}$ & $v=1-1, J=80-79$ & 1433.086 & 209.194 & $7.36 \mathrm{e}-14$ & yes \\
\hline${ }^{28} \mathrm{Si}^{32} \mathrm{~S}$ & $v=1-1, J=81-80$ & 1450.688 & 206.655 & $2.50 \mathrm{e}-14$ & no \\
\hline${ }^{28} \mathrm{Si}^{32} \mathrm{~S}$ & $v=1-1, J=82-81$ & 1468.278 & 204.180 & $2.65 e-14$ & yes \\
\hline${ }^{28} \mathrm{Si}^{32} \mathrm{~S}$ & $v=1-1, J=83-82$ & 1485.857 & 201.764 & $2.78 \mathrm{e}-14$ & yes \\
\hline${ }^{28} \mathrm{Si}^{32} \mathrm{~S}$ & $v=1-1, J=84-83$ & 1503.423 & 199.407 & $2.93 e-14$ & yes \\
\hline${ }^{28} \mathrm{Si}^{32} \mathrm{~S}$ & $v=1-1, J=85-84$ & 1520.978 & 197.105 & $7.32 \mathrm{e}-14$ & yes \\
\hline${ }^{28} \mathrm{Si}^{32} \mathrm{~S}$ & $v=1-1, J=86-85$ & 1538.520 & 194.858 & $3.18 \mathrm{e}-14$ & yes \\
\hline${ }^{28} \mathrm{Si}^{32} \mathrm{~S}$ & $v=1-1, J=87-86$ & 1556.050 & 192.662 & $3.18 \mathrm{e}-14$ & no \\
\hline${ }^{28} \mathrm{Si}^{32} \mathrm{~S}$ & $v=1-1, J=88-87$ & 1573.567 & 190.518 & $7.40 \mathrm{e}-14$ & no \\
\hline${ }^{28} \mathrm{Si}^{32} \mathrm{~S}$ & $v=1-1, J=89-88$ & 1591.072 & 188.422 & $7.36 \mathrm{e}-14$ & yes \\
\hline${ }^{28} \mathrm{Si}^{32} \mathrm{~S}$ & $v=1-1, J=90-89$ & 1608.564 & 186.373 & $7.34 \mathrm{e}-14$ & no \\
\hline${ }^{28} \mathrm{Si}^{32} \mathrm{~S}$ & $v=1-1, J=91-90$ & 1626.043 & 184.369 & $7.26 \mathrm{e}-14$ & no \\
\hline${ }^{28} \mathrm{Si}^{32} \mathrm{~S}$ & $v=1-1, J=92-91$ & 1643.509 & 182.410 & $7.13 \mathrm{e}-14$ & yes \\
\hline${ }^{28} \mathrm{Si}^{32} \mathrm{~S}$ & $v=1-1, J=93-92$ & 1660.962 & 180.493 & $7.07 \mathrm{e}-14$ & yes \\
\hline${ }^{28} \mathrm{Si}^{32} \mathrm{~S}$ & $v=1-1, J=94-93$ & 1678.402 & 178.618 & $7.18 \mathrm{e}-14$ & no \\
\hline${ }^{28} \mathrm{Si}^{32} \mathrm{~S}$ & $v=1-1, J=95-94$ & 1695.828 & 176.782 & $6.93 e-14$ & no \\
\hline${ }^{28} \mathrm{Si}^{32} \mathrm{~S}$ & $v=1-1, J=96-95$ & 1713.240 & 174.986 & $6.97 \mathrm{e}-14$ & no \\
\hline${ }^{28} \mathrm{Si}^{32} \mathrm{~S}$ & $v=1-1, J=97-96$ & 1730.639 & 173.226 & $6.67 e-14$ & yes \\
\hline${ }^{28} \mathrm{Si}^{32} \mathrm{~S}$ & $v=1-1, J=98-97$ & 1748.024 & 171.504 & $6.84 \mathrm{e}-14$ & no \\
\hline${ }^{28} \mathrm{Si}^{32} \mathrm{~S}$ & $v=1-1, J=99-98$ & 1765.394 & 169.816 & $6.79 \mathrm{e}-14$ & yes \\
\hline${ }^{28} \mathrm{Si}^{32} \mathrm{~S}$ & $v=1-1, J=100-99$ & 1782.751 & 168.163 & $6.66 \mathrm{e}-14$ & yes \\
\hline${ }^{28} \mathrm{Si}^{32} \mathrm{~S}$ & $v=1-1, J=101-100$ & 1800.093 & 166.543 & $6.38 \mathrm{e}-14$ & no \\
\hline${ }^{28} \mathrm{Si}^{32} \mathrm{~S}$ & $v=1-1, J=102-101$ & 1817.420 & 164.955 & $6.52 \mathrm{e}-14$ & no \\
\hline${ }^{28} \mathrm{Si}^{32} \mathrm{~S}$ & $v=1-1, J=103-102$ & 1834.733 & 163.398 & $6.21 \mathrm{e}-14$ & no \\
\hline${ }^{28} \mathrm{Si}^{32} \mathrm{~S}$ & $v=1-1, J=104-103$ & 1852.032 & 161.872 & $6.32 \mathrm{e}-14$ & yes \\
\hline${ }^{28} \mathrm{Si}^{32} \mathrm{~S}$ & $v=1-1, J=105-104$ & 1869.315 & 160.376 & $6.08 \mathrm{e}-14$ & yes \\
\hline${ }^{28} \mathrm{Si}^{32} \mathrm{~S}$ & $v=1-1, J=106-105$ & 1886.583 & 158.908 & $6.00 \mathrm{e}-14$ & yes \\
\hline${ }^{28} \mathrm{Si}^{32} \mathrm{~S}$ & $v=1-1, J=107-106$ & 1903.836 & 157.468 & $6.00 \mathrm{e}-14$ & yes \\
\hline${ }^{28} \mathrm{Si}^{32} \mathrm{~S}$ & $v=1-1, J=108-107$ & 1921.074 & 156.055 & $5.87 \mathrm{e}-14$ & yes \\
\hline${ }^{28} \mathrm{Si}^{32} \mathrm{~S}$ & $v=1-1, J=109-108$ & 1938.296 & 154.668 & $5.77 \mathrm{e}-14$ & yes \\
\hline
\end{tabular}

Notes. The integrated flux values in the fifth column are for the model predictions as shown in Fig. 1. The last column indicates whether a line is blended strongly with another line. 